ApJ, to be submitted, September 13, 2016

\title{
Detecting Relativistic X-ray Jets in High-Redshift Quasars
}

\author{
Kathryn McKeough ${ }^{1}$, Aneta Siemiginowska ${ }^{2}$, C. C. Cheung ${ }^{3}$, \\ Lukasz Stawarz ${ }^{4}$, Vinay L. Kashyap ${ }^{2}$, \\ Nathan Stein ${ }^{5}$, Vasileios Stampoulis ${ }^{6}$, David A. van Dyk $^{6}$, \\ J. F. C. Wardle ${ }^{7}$, N. P. Lee ${ }^{2}$, D. E. Harris ${ }^{2 *}$, D. A. Schwartz ${ }^{2}$, Davide Donato ${ }^{8}$, \\ Laura Maraschi ${ }^{9}$ and Fabrizio Tavecchio ${ }^{9}$. \\ kathrynmckeough@g.harvard.edu
}

\begin{abstract}
We analyze Chandra X-ray images of a sample of 11 quasars that are known to contain kiloparsec scale radio jets. The sample consists of five high-redshift $(z \geq$ 3.6) flat-spectrum radio quasars, and six intermediate redshift $(2.1<z<2.9)$ quasars. The dataset includes four sources with integrated steep radio spectra and seven with flat radio spectra. A total of 25 radio jet features are present in this sample. We apply a Bayesian multi-scale image reconstruction method to detect and measure the X-ray emission from the jets. We compute deviations from a baseline model that does not include the jet, and compare observed X-ray
\end{abstract}

\footnotetext{
${ }^{1}$ Department of Statistics, Harvard University, Cambridge, MA 02138

${ }^{2}$ Harvard-Smithsonian Center for Astrophysics, 60 Garden St., Cambridge, MA 02138, USA

${ }^{3}$ Space Science Division, Naval Research Laboratory, Washington, DC 20375-5352, USA

${ }^{4}$ Astronomical Observatory, Jagiellonian University, ul. Orla 171, 30-244, Kraków, Poland

${ }^{5}$ Department of Statistics, The Wharton School, University of Pennsylvania, 400 Jon M. Huntsman Hall, 3730 Walnut Street, Philadelphia, PA 19104-6340, USA

${ }^{6}$ Statistics Section, Imperial College London, Huxley Building, South Kensington Campus, London SW7,

${ }^{7}$ Department of Physics, MS 057, Brandeis University, Waltham, MA 02454, USA

${ }^{8}$ CRESST and Astroparticle Physics Laboratory NASA/GSFC, Greenbelt, MD 20771, USA

${ }^{9}$ INAF Osservatorio Astronomico di Brera, via Brera 28, 20124, Milano, Italy

${ }^{*}$ Deceased formally from Harvard-Smithsonian Center for Astrophysics
} UK 
images with those computed with simulated images where no jet features exist. This allows us to compute $p$-value upper bounds on the significance that an $\mathrm{X}$ ray jet is detected in a pre-determined region of interest. We detected 12 of the features unambiguously, and an additional 6 marginally. We also find residual emission in the cores of 3 quasars and in the background of 1 quasar that suggest the existence of unresolved X-ray jets. The dependence of the X-ray to radio luminosity ratio on redshift is a potential diagnostic of the emission mechanism, since the inverse Compton scattering of cosmic microwave background photons (IC/CMB) is thought to be redshift dependent, whereas in synchrotron models no clear redshift dependence is expected. We find that the high-redshift jets have X-ray to radio flux ratios that are marginally inconsistent with those from lower redshifts, suggesting that either the X-ray emissions is due to the IC/CMB rather than the synchrotron process, or that high redshift jets are qualitatively different.

Subject headings: Galaxies: active — galaxies: jets — quasars: general — radiation mechanisms: non-thermal — radio continuum: galaxies — X-rays: galaxies

\section{Introduction}

Jets in active galactic nuclei transfer the energy generated by the central supermassive black hole $(\mathrm{SMBH})$ to large $(>100 \mathrm{kpc})$ distances. The impact of jets on the environment contributes to the formation and evolution of structures in the early Universe (Croton et al. 2006). The innermost jets (parsec-scales or smaller) of radio-loud quasars are highly relativistic and their observed radiation can be Doppler amplified when observed at small angles to the line of sight. These jets can be bright at high-energies and thus can provide interesting observational probes of the state of the SMBH activity (Begelman et al. 1984), but they remain spatially unresolved in X-rays and $\gamma$-rays.

Large scale X-ray jets span distances out to hundreds of kiloparsecs away from the SMBH and encode the history of SMBH activity during the jet's lifetime (a few Myrs). Their X-ray emission can be resolved with the Chandra X-ray Observatory. The number of such X-ray jets has significantly increased since the launch of Chandra in 1999, but it is still relatively small in comparison to the number of known quasars. There are about 100 large scale Xray jets detected to date and only a few of them have good quality X-ray morphology data (Massaro et al. 2011). Though a direct connection between SMBH activity and the existence of kpc-scale jets is ambiguous, and the X-ray emission mechanism is not well understood, high-redshift $(z>3)$ jets could potentially establish the dominant energy environment in 
the early Universe. Such jets probe the physics of the earliest (first $\sim 2$ Gyr of the Universe in the quasars studied) actively accreting SMBH systems and are also interesting for other reasons. For instance, the ambient medium in these high-redshift galaxies is probably higher than that of lower redshift galaxies (e.g., De Young 2006) and this may manifest itself in jets with different morphologies, with increased energy dissipation, or with the jets being slower in general than their lower-redshift counterparts.

The X-ray radiation could be attributed to either synchrotron emission by highly relativistic electrons (Lorentz factors of $\gamma \sim 10^{7}-10^{8}$ ) in relatively strong magnetic fields, or inverse Compton scattering of the cosmic microwave background (IC/CMB) photons off the low energy $\left(\gamma \sim 10^{3}\right)$ large-scale jet particles (for a review see Harris \& Krawczynski 2006). In the simplest scenario, such models have diverging predictions at high redshift. Specifically, we expect a strong redshift dependence in the X-ray-to-radio- energy flux ratio, $\rho_{x r}=\frac{F_{\mathbf{x}}}{\nu_{\mathrm{r}} f_{\mathrm{r}}}$, where the radio fluxes are given at the observed frequency, and the X-ray fluxes are modeled over the energy range $0.5-7 \mathrm{keV}$ (Massaro et al. 2011). Typically, $\rho_{x r} \propto U_{\mathrm{CMB}} \propto(1+z)^{4}$ for IC/CMB, whereas in synchrotron models, we do not expect a strong dependence $1^{1} \rho_{x r} \propto(1+z)^{0}$. Below we compare the predictions of these two models for the highest-redshift relativistic jets.

Most Chandra studies of quasar jets have so far targeted known arcsecond-scale radio jets (e.g., Sambruna et al. 2004; Marshall et al. 2011), as most known examples are at $z$ $\lesssim 2$ (Bridle \& Perley 1984; Liu \& Zhang 2002). At the time our program began, there were two high- $z$ quasars with kpc-scale X-ray jets: GB $1508+5714$ at $z=4.3$ (Siemiginowska et al. 2003; Yuan et al. 2003; Cheung 2004) and 1745+624 at $z=3.9$ (Cheung et al. 2006). They were observed to have large $\rho_{x r}$ values consistent with the IC/CMB model (Schwartz 2002; Cheung 2004), although the small number of high- $z$ detections precluded any definitive statements (Kataoka \& Stawarz 2005; Cheung et al. 2006).

We have therefore obtained Chandra X-ray observations of an additional four highredshift $(z>3.6$; GB 1508+5714 was previously analyzed) and six intermediate-redshift $(2 \leq z \leq 3)$ quasars with known radio jets. The highest redshift X-ray and radio jet discovered in the sample studied (at $z=4.72$, in GB 1428+4214 (1428+422)) was presented and discussed in detail by Cheung et al. (2012). New and archival arcsecond-resolution NRAO 2 imaging observations of these quasars are also presented.

\footnotetext{
${ }^{1}$ The energies of the synchrotron emitting electrons are different in the observed radio and X-ray spectra, $\gamma_{\mathrm{r}} \sim 10^{3}$ vs. $\gamma_{\mathrm{x}} \sim 10^{7}$ and these electrons may originate in the same population or two different populations. Therefore, there could be some weak redshift dependence in the synchrotron model.

${ }^{2}$ The National Radio Astronomy Observatory is operated by Associated Universities, Inc. under a coop-
} 
The small number of X-ray photon counts observed from jets relative to their corresponding quasar cores means that detecting X-ray jets is inherently challenging. Statistically, we must test the hypothesis that a baseline model of the quasar core and a flat background, without a jet, is insufficient to explain the observed data. We do this test using a multi-scale

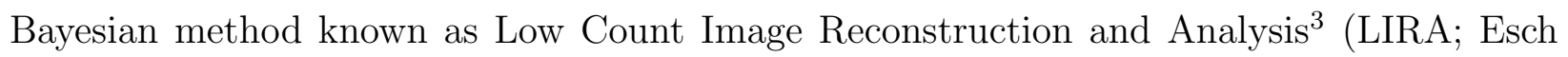
et al. 2004; Connors \& van Dyk 2007). The algorithm models the residual as a multi-scale component, and generates a series of images that capture the emission that may be present in excess of the baseline model. We can then compute a $p$-value $4^{4}$ by generating a series of Monte Carlo simulations of images under the baseline model and fitting each of these simulated images using LIRA. Stein et al. (2015) (hereafter Paper I) show how an upper-bound on the $p$-value can be computed with a small number of MCMC replicates. We are interested in detecting whether X-ray jets exist in regions where jets were previously observed in the radio band. In this paper, we will not consider X-ray detections without a corresponding radio emission (such a detection of a jet that was recently reported by Simionescu et al. (2016)) when matching our results to the IC/CMB or synchrotron emission model. We run LIRA to detect jets in pre-defined regions of an X-ray image. Using the jets detected in $\mathrm{X}$-rays we are able to observe how $\rho_{x r}$ is dependent on redshift and whether it matches the predictions of the IC/CMB or synchrotron emission model.

Section 2 describes the sample selection and initial processing of the X-ray and corresponding radio observations. Section 3 outlines how LIRA is used to find evidence that a jet exists in a region where one is observed in radio imaging. Section 4 elaborates on the results of the image analysis methods when applied to the new X-ray observations. Section 5 gives a final description of our results in context and we summarize our results in Section 6 .

erative agreement with the National Science Foundation.

${ }^{3}$ LIRA is implemented as a package for the $\mathrm{R}$ statistical programming language (r-project.org) that is available for downloading and use at github.com/astrostat/LIRA.

${ }^{4}$ Formally, a $p$-value is the probability that the baseline null hypothesis can generate a value for the test statistic as large as that which is observed. In this case, it defines the likelihood that a given intensity can be obtained under the assumption that the baseline model is the truth. That is, when the $p$-value is small, the chances that the feature under consideration can be attributed to a fluctuation is small. This allows us to reject the null hypothesis when this probability falls below a pre-defined threshold. Note, however, that it should never be interpreted as a measure of the probability that the alternate hypothesis is true, nor, if the null cannot be rejected, as a measure of the probability that the null hypothesis is true (Wasserstein \& Lazar 2016). 


\section{Sample Selection and Observations}

Compilations of known radio jets (Bridle \& Perley 1984; Liu \& Zhang 2002) show very few examples with kpc-scale extensions at $z>3$. To increase the number of known radio jets, we carried out a VLA survey of the highest redshift $(z>3.4)$ quasars and searched for extended radio emission (Cheung et al. 2005, 2008). The sample sources are the brightest ( $\gtrsim 100 \mathrm{mJy}$ at 1.4 and/or $5 \mathrm{GHz}$ to facilitate ease of radio mapping), flat-spectrum radio sources catalogued by NED. The flat spectra is a good proxy for high beaming and our high redshift sample is representative of a general radio-loud quasars population.

Four flat-spectrum radio jets from the $z>3.4$ radio sample were observed in a Chandra AO8 program (PI: Cheung) from 2007 Jan - June (ObsIDs: 7871-7874): 1239+376 ( $z=3.82)$, $1754+676(z=3.60), 1418-064(z=3.69), 1428+422(z=4.72)$, in addition to one quasar at $z=2.10(0833+585$; ObsID 7870) which had a known long radio jet (Murphy et al. 1993). The jets have minimum projected lengths of $2.5^{\prime \prime}$ and up to $\sim 15^{\prime \prime}$ for the $z=2.1$ case (Table 1), so their X-ray counterparts are easily separable from the bright nucleus in the Chandra images. The exposures of $\sim 3.8-11 \mathrm{ks}$ were tailored to the radio jet brightness and redshifts.

We also undertook a Chandra survey of an intermediate redshift $(2 \leq z \leq 3)$ sample of five sources in AO10 (PI: Sambruna) from Jan - May 2009 (ObsIDs: 10307-10311) with exposures set to $\sim 20 \mathrm{ks}$. These radio jets were selected to have lengths greater than $2.5^{\prime \prime}$ from the list of Liu \& Zhang (2002). In order to sample a range of jet orientations with respect to our line of sight, the selected targets have a range of radio core to extended flux

ratios indicating both core-dominated and lobe-dominated radio sources. A summary of the basic properties of the sources are recorded in Table 1 .

\section{1. $\quad$ Very Large Array Observations}

The details of the VLA observations for the entire sample are shown in Table 2. These radio jets form the largest sample of the radio jets at high redshift that were observed with Chandra. We analyzed the archival data and selected the observations with the best astrometry for this project. Portions of these radio data were presented in Gobeille (2011) and Gobeille et al. (2014).

The standard calibration was applied using AIPS (Bridle \& Greisen 1994) with scans of primary calibrators, 3C 48 or 3C 286, used to set the flux density scales. The data was then exported to the Caltech DIFMAP package (Shepherd et al. 1994) for self-calibration and imaging. 
The radio jets in 1418-064, 1428+422, and 1754+676 were discovered in VLA $1.4 \mathrm{GHz}$ A-configuration images from Oct - Dec 2004 (program AC755) and discussed in Cheung et al. (2005). We utilized the archival VLA snapshots of 1239+376 (Taylor et al. 1996) and 0833+585 (Murphy et al. 1993). The details of the 1428+422 observations are described in Cheung et al. (2012) but are included in Table 2 for completeness.

The quasar 1418-064 has a hint of a radio extension in the VLA $1.4 \mathrm{GHz}$ observation thus we obtained new A-array data at $5 \mathrm{GHz}$ (July 27-28, 2007; program S8723) of this object which revealed more detail in the radio jet.

\subsection{Chandra X-ray Observations.}

All X-ray observations were made with the Chandra (Weisskopf et al. 2002; Schwartz 2014) ACIS-S back-illuminated CCD. The Chandra observations are listed in Table 3 . We used the nominal aimpoint of the ACIS-S3 chip and a 1/8th subarray mode (0.4s frame time) in order to mitigate pileup of the nucleus. Roll angles were selected to place a possible charge transfer streak from the X-ray nucleus away from the jet position angles. We reprocessed the data by using chandra_repro script in CIAO (Fruscione et al. 2006) and assigned the calibration available in CALDB v4.6. We inspected the data for any possible background flares and concluded that the data were not affected by flares. The background level in our short observations was low and detections of the quasar emission and some jet features are highly significant. We only use the X-ray events with energies between $0.5-7 \mathrm{keV}$ in the spectral and image analysis described below.

The X-ray nuclei are all clearly detected and the Chandra positions (peaks) are within the $0.6^{\prime \prime}$ (90\% pointing accuracy Weisskopf et al. (2002)) of the radio positions (Table 1). We use the method described below for image analysis and detections of any features outside the core.

All spectral modeling was performed in Sherpa (Freeman et al. 2001) CIAO version v4.6. We extracted the spectra and created response files for each observation using CIAO tools. We used the Nelder-Mead optimization algorithm and Cash likelihood statistics appropriate for low counts data and fit the spectra in Sherpa. We assumed an absorbed power law model for each identified feature with the absorption column fixed at the appropriate Galactic value (Colden; Stark et al. (1992)). The photon index of the power law and the normalization were fit. For the cores, we also fit a model which included an additional intrinsic absorption component at the quasar redshift. 


\section{X-ray Image Analysis Methods}

\subsection{Initial Analysis}

For each Chandra observation we generate an image centered on the quasar rebinned to a pixel size of $0.246^{\prime \prime}$ (half of the native ACIS pixel size.) For sources with a radio jet located at $<7^{\prime \prime}$ from the quasar core we use $64 \times 64$ pixel $\left(15.7^{\prime \prime} \times 15.7^{\prime \prime}\right)$ X-ray images. For the three sources with larger radio structures we use $128 \times 128$ pixels $\left(31.5^{\prime \prime} \times 31.5^{\prime \prime}\right)$ images.

We employ SAOtrace $5^{5}$ to generate Chandra point-spread functions (PSFs) for each observation, constructing the PSF with parameters appropriate to the quasar location. Since $\mathrm{X}$-ray PSFs are energy dependent, the raytraced PSFs include spectral models derived from fitting the spectrum of each quasar. We use the same pixel size as in the X-ray image when binning the PSF.

We select regions of interest (ROI) for the quasar core and their jets using existing radio images, extracting spectra and response files using specextract. The radio images appear in panel (a) of Figure 1 and of Figures 514 in Appendix B and the ROIs are overlaid on Chandra ACIS-S images in panel (b) of each figure. The circular quasar regions are marked ' $Q$ ' and are centered on the radio core with the intent to encapsulate extended regions around the core, and the elliptical ROIs are associated with jet features and are numbered. The complementary region is the remainder of the image that excludes both the quasar and jet regions and are marked 'C'. The number of ROIs vary from one quasar to another. Spectra are extracted from these regions and fit with an absorbed power-law model. The results are in Table 6.

${ }^{5}$ CXC Optics: http://cxc.harvard.edu/cal/Hrma/SAOTrace.html 
(a)
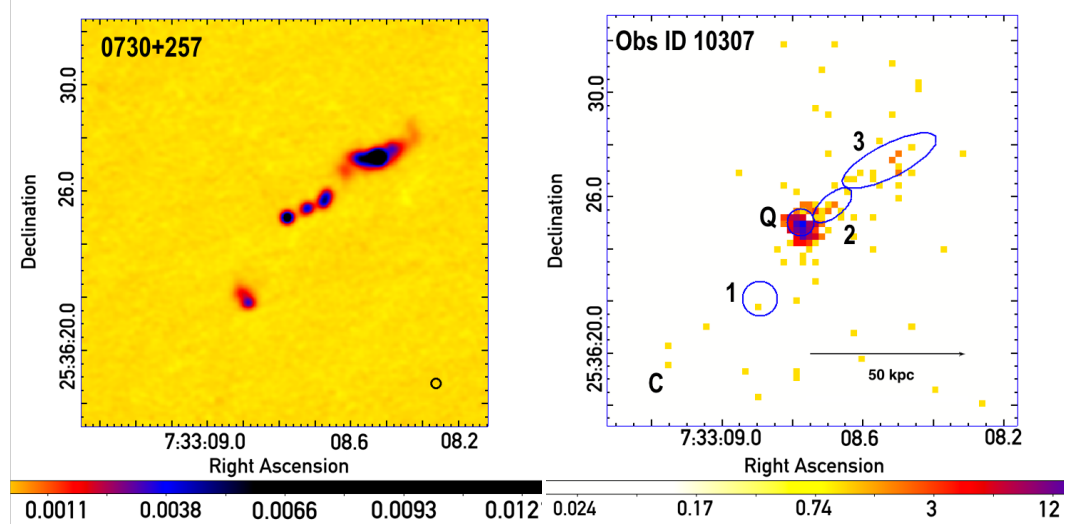

(d)
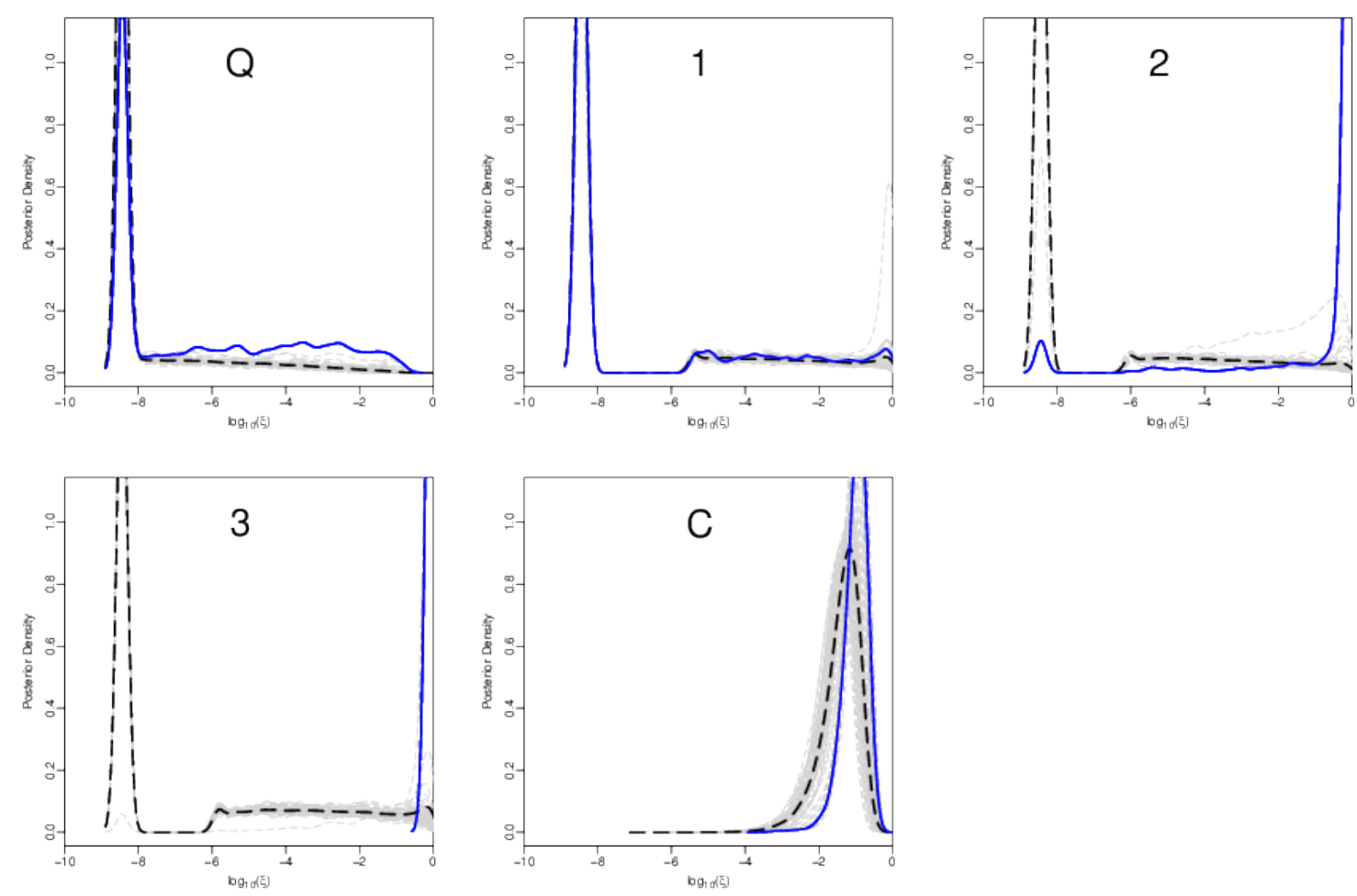

(c)

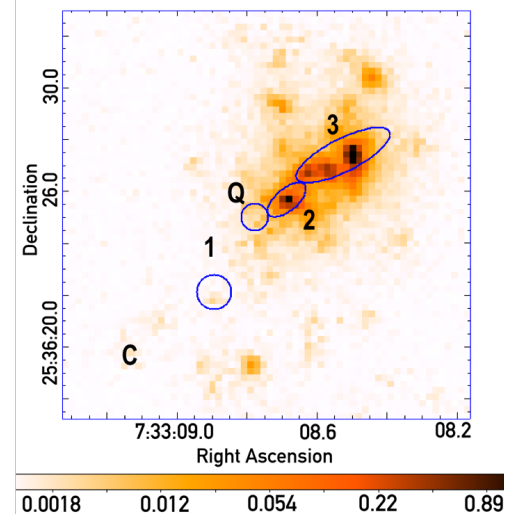

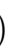




\subsection{Image Reconstruction using LIRA}

Detection of an X-ray jet is particularly challenging in part because it is a faint structure located near a much stronger quasar core. The Bayesian multi-scale fitting method LIRA is well suited to this challenge. An efficient feature detection method is described in Paper I as an addendum to the multi-scale fitting. Section 2 of Paper I gives full details of the statistical models used by LIRA, which we briefly review here. We consider square images of $n=2^{d} \times 2^{d}$ pixels, where $d$ is an integer (for typical cases considered here, $d=6$ or 7 ). We denote the counts in each pixel by $\boldsymbol{y}=\left(y_{i}, i=1 \ldots n\right)$, with the total number of photon counts, $N=\sum_{i} y_{i}$. The image is then modeled as the sum of a known baseline component, here representing the quasar core and background, and an added multi-scale component, here representing the jet. The counts observed in detector pixel $i$ are modeled as

$$
y_{i} \stackrel{\text { indep }}{\sim} \text { Poisson }\left(\sum_{j=1}^{n} P_{j i} A_{j}\left(\tau_{0} \Lambda_{0 j}+\tau_{1} \Lambda_{1 j}\right)\right),
$$

where $\left(P_{j i}, i=1 \ldots n, j=1 \ldots n\right)$ is the PSF, representing the probability that a photon arriving from the direction represented by pixel $j$ is recorded in pixel $i,\left(A_{j}, j=1 \ldots n\right)$ is the exposure map, representing the efficiency with which incoming photons at pixel $j$ will be recorded, $\tau_{1}$ is the total intensity of the added component, and $\tau_{0}$ is the total intensity of the baseline component. The quantities $\Lambda_{k}=\left(\Lambda_{k 1}, \ldots, \Lambda_{k n}\right)$ (for $\left.k=0,1\right)$ are the proportions of $\tau_{k}$ in the individual pixels. Note that this represents a general model that is applied to the full image. For ROIs smaller than the full image (see below), we first apply the model

in Equation 1 to the full image, then recompute $\tau_{k}=\sum_{j \in \mathrm{ROI}} \mu_{k j}$, where $\mu_{k j}=\left(\tau_{k} \Lambda_{k j}\right)$ are the inferred intensities in each pixel.

Paper I describes how LIRA uses a flexible multi-scale model in order to accommodate added structure, such as a jet, in the quasar image. LIRA combines the likelihood given in Equation 11 with prior distributions to formulate a posterior distribution for $\tau_{0}, \tau_{1}$, and $\Lambda_{1}$ and uses MCMC to provide a Monte Carlo sample from this posterior distribution.

\subsection{Using LIRA to Quantify Evidence for Extended Jet Emission}

LIRA uses a binned Chandra image, a ray traced Chandra PSF, and a composite Gaussian plus constant baseline model representing the quasar core and background respectively. The MCMC sampler within LIRA returns a sequence of simulated images of the residual multi-scale component, including any non-Poisson deviations from the baseline model, which in the cases studied, would indicate a jet emanating from the X-ray quasar.. If the Chandra 
image is consistent with the baseline model (quasar core + background), the fitted image of the multi-scale component contains only random fluctuations. On the other hand, any $\mathrm{X}$-ray emission not described by the baseline component appears as a systematic deviation from this noise. Substantial deviations can be taken as evidence for structures beyond the baseline component in the Chandra image.

Section 3 of Paper I describes a formal statistical hypothesis test for the presence of structure (e.g., a quasar jet) in the added multi-scale component and how we can compute an upper bound on the $p$-value for this test. This involves Monte Carlo evaluation of the behavior of the fitted multi-scale component (which describes the deviation from the baseline image) when there is no actual structure in the X-ray image beyond the baseline model (i.e., when there is no jet). Quantitatively, this involves using a test statistic to efficiently compute an upper bound on the $p$-value. Here we outline this method; further details can be found in Paper I.

To start, for each observed image we simulate 50 replicate images under a baseline-only model using Sherpa's fake function. The baseline model is composed of a 2D Gaussian for the quasar core on top of constant background and set to the best-fit values determined by fitting a 2D model, psf*gauss2d+const2d, to the observed X-ray image. For each quasar, we run LIRA 6] and discard the first 1000 on the Chandra images as well as each of its 50 replicate baseline-only simulated images. The image of the deviation from the baseline when fit to the simulated images contain only random fluctuations, while those fit to the Chandra images contain estimates of any structure beyond the baseline model. A jet is considered detected if the signal in the region of interest is stronger than is likely to occur through random fluctuations under the baseline-only model. Thus, we must compare the fitted deviations from the baseline of the Chandra observation with the corresponding 50 fitted deviations of the baseline-only simulations.

This is done quantitatively through a novel test statistic that compares the expected photon counts due to the baseline and added components, i.e., it compares $\tau_{0}$ with $\tau_{1}$ in Equation 1. The test statistic is unusual in that it is a posterior probability, namely,

$$
T_{c}(\boldsymbol{y})=\operatorname{Pr}(\xi \geq c \mid \boldsymbol{y})
$$

given a threshold $c$ where

$$
\xi=\tau_{1} /\left(\tau_{1}+\tau_{0}\right)
$$

\footnotetext{
${ }^{6}$ We only use LIRA iterations that are obtained after an initial burn in of the MCMC sampler. In all cases, we obtain 2000 iterations for each run
} 
is the proportion of expected counts due to the added structure. If there is no structure in the added component (e.g., no quasar jet), $\xi$ and thus $T_{c}(\boldsymbol{y})$ are close to zero; as the intensity of the added component grows, $\xi$ and $T_{c}(\boldsymbol{y})$ tend toward one. In this regard, $T_{c}(\boldsymbol{y})$ is a useful statistic for testing for added structure, i.e., for the quasar jet.

\section{Results}

\subsection{Quasars}

The quasar nuclei in our sample were observed by Chandra with photon counts ranging from 64 to 5500. We obtained a relatively good spectrum for each quasar core, and fit a power-law with a fixed Galactic absorption model to each spectrum using Sherpa (Freeman et al. 2001). We also considered an additional intrinsic absorption component (xsphabs $(\mathrm{Gal}) *$ xszphabs $\left(z_{q s o}\right) *$ powlaw1d). The results of our spectral fits are shown in Table 4. An intrinsic absorption is required in four quasars $(0730+257,1218+112,1834+612$, and $1428+422$ ), with absorption columns of $\approx 10^{22} \mathrm{~cm}^{-2}$. For the remaining seven quasars we obtained upper limits. The photon indices for the power law model range between $\Gamma_{X}=1.35$ and $\Gamma_{X}=2.26$ with an average of $\Gamma_{X}=1.7 \pm 0.2$, consistent with estimates for large samples of radio-quiet and radio-loud quasars (Kelly et al. 2007; Young et al. 2009, Lanzuisi et al. 2013). All the quasars are X-ray luminous, with the luminosities in $2-10 \mathrm{keV}$ energy band

exceeding $2.5 \times 10^{45} \mathrm{erg} \mathrm{s}^{-1}$. The most luminous quasar in our sample $(1428+422)$ has a $2-10 \mathrm{keV}$ luminosity of $4.0 \pm 0.2 \times 10^{47} \mathrm{erg} \mathrm{s}^{-1}$.

\subsection{Jets}

We applied the method presented in Section 3 to our sample of 11 high-redshift quasars. The posterior mean of $\tau_{1} \Lambda_{1}$ is plotted in panel (c) of Figure 1 and of Figures 5 - 14 in Appendix B. These represent the fitted multi-scale structure added to the baseline, i.e., the jets. In order to assess the significance of the X-ray emission in each ROI and claim a detection, we calculate an upper bound on the $p$-value (with an upper tail probability of $\gamma=0.005$, see section 3.4 of Paper I). This requires us to post-process the LIRA MCMC posterior sample to obtain a posterior sample of $\xi$ for each ROI. Panel (d) of Figure 1 and of Figures 514 in Appendix B display the smoothed distributions of $\xi$ for each ROI for the data (solid blue line), and the averaged distributions of $\xi$ over the same ROI for the null simulations (dashed black). The distributions of $\xi$ for each of the 50 simulated null images are shown as grey lines. We expect the observed distribution to depart from the distributions based on the null 
simulations if there is significantly more X-ray emission than expected under the baseline model.

We set a threshold of $p=0.01$ to determine the existence of a significant feature. That is, if the upper bound on the $p$-value for the distribution of $\xi$ in an ROI is $\leq 0.01$, we take that to be sufficient evidence for X-ray emission associated with a jet feature in the ROI. Table 5 lists the upper bounds on the $p$-value for all the ROIs, showing that 16 features (12 jet features, 3 cores and 1 complementary region) have a $p$-values less than 0.01 . There are an additional 7 ROIs with an upper bound on the $p$-value between 0.01 and 0.02 which we consider marginal detections. Thus, in total we list 23 significant X-ray features associated with high redshift jets including the corresponding cores and complementary regions. In all these cases the distributions of $\xi$ based on the Chandra data are skewed to the right compared to the distributions based on the null simulations.

There are three ROIs associated with the quasar cores (region Q for sources $1508+5714$, $1428+422$ and $1834+612$ ) for which the upper bound on the $p$-value is lower than our predefined $p$-value threshold of $\alpha=0.01$. This indicates the detection of the X-ray emission in excess of a point source assumed for the spatial model of the quasar core emission. In general, all the images representing the deviation from the baseline model (panel (c) of Figure 1 and of Figures 5 14 in Appendix B ) show indications of excess counts in the core ROIs in all the sources; we attribute these to an imperfect knowledge of the true telescope PSF, with the apparent deviations reflecting the uncertainties in the adopted PSF. However, only in three quasars are they significant, signaling a departure from the baseline model. Note that this also shows that our threshold for detection is stringent enough to avoid detecting spurious features that may arise due to uncertainties in the PSF.

Note that the in the case of $1508+5714$, a significant secondary structure in the complementary region ' $\mathrm{C}$ ' is seen, implying that not all the $\mathrm{X}$-ray features were captured by the radio-based regions. This object has a deep Chandra observation of about $90 \mathrm{ksec}$, the longest one in our sample, and it is not unusual to find such structure in long observations.

Our X-ray jets detection rate - 9 sources with detected jets out of 11 with known jets - is similar to the one reported for the lower redshift sources $(\sim 70 \%$ detection rates for exposures of $\sim 5-10 \mathrm{ksec}$; Sambruna et al. (2004); Marshall et al. (2011)). 


\section{Discussion}

\subsection{X-ray Morphology}

The posterior mean images $\tau_{1} \Lambda_{1}$ representing deviations from the baseline model of the Chandra data (panel (c) of Figure 1 and of Figures 514 in Appendix B provide a view of the X-ray morphology with the quasar core removed. However, not all the structures seen in these images are significant, since many features could be attributed to statistical fluctuations. We have developed a method (Paper I; see also Section 2 above) to assess the significance of the emission for well-defined ROIs. Note that these ROIs must be set prior to the analysis and cannot be deduced from the LIRA output, since doing so would increase the false detection rate. We adopt regions based on the locations of the radio jet features, but note that the jet X-ray emission may not always be spatially coincidental with the radio emission (Schwartz 2002), nor even have a radio counterpart (Jorstad \& Marscher 2004; Simionescu et al. 2016). Offsets between the radio and X-ray peaks in the jet features have been reported (e.g. Siemiginowska et al. 2007; Worrall 2009). For instance, in 1754+676 (ObsID 7872), the X-ray jet is not detected, but the image showing deviations from the baseline (see panel (c) of Figure 11) suggests the existence of an emission feature between the quasar and the radio jet region. A longer Chandra observation is necessary to confirm this emission. In another source 0805+046 (ObsID 10308), the image showing deviations from the baseline (panel (c) of Figure 5) displays considerable emission outside the narrow radio jet, suggesting a more complex X-ray morphology.

We used the complementary regions to assess the possibility of unexpected X-ray emission present outside the pre-defined regions. In all sources but $1508+5714$, we do not find a strong indication that such emission is present, though the complementary regions cover a large area and thus statistical tests have relatively low power to detect smaller compact structures. Future studies of the X-ray morphology in the vicinity of this source is required for understanding the origin of this emission.

The $p$-value upper bound test relies on the test regions being pre-defined. This is done in order to avoid the loss of power in the test that arises when multiple hypotheses are tested. We thus take the regions directly from the radio data and do not optimize the regions based on the X-ray data. This could result in the size of location of the regions to be slightly misaligned, reducing the significance of detection. For example, ROI 1 in source 1428+422 (ObsID 7874) is a marginal detection with an upper bound on the $p$-value of 0.010 , but decreasing the region size from 91 to 77 pixels, an arguably better fit for this object in radio, results in an improvement in the $p$-value upper bound to 0.009 , which crosses the threshold into a significant detection. Areas of deviation from the baseline may be difficult to detect 
in a large region or a region may not encompass all of the relevant area of the image, and the better the region fits an area with deviation from the baseline, the lower the nominal $p$-value is. But when large numbers of regions are tested, the $p$-value threshold must be reduced correspondingly in order to prevent false claims of detections due to fluctuations. For instance, if the $\xi$ in 20 regions (say) of radii stepping from 75 to 95 pixels are tested, the appropriate threshold of $\alpha$ must be reduced by a factor of 20 , to $\alpha=0.0005$, to maintain the same level of significance. We emphasize that ROI selection must be consistent across the analysis, and must be defined before applying the significance test. We also note that we apply the test to a total of 47 ROIs, so we expect at most 1 false positive amongst the claimed significant and marginal detections at the significance threshold of $\alpha<0.02$.

As noted above, our current method of region selection depends on radio data. In the future, we plan to develop methods that are independent of the radio (or other wavebands) selections and autonomously generate regions that adaptively fit the deviations from the baseline in the LIRA output. Such a method is needed since the X-ray emission does not always follow the radio closely. Naturally, any such method will trade-off ROI optimization for the statistical power of the detection routine.

\subsection{Redshift Dependence in Large-Scale X-ray/Radio Emission}

The origin of X-ray jet emission is still under debate. An early hint at the advantage of studying high-redshift jets came from the $z=4.3$ quasar $1508+5714$ (Siemiginowska et al. 2003; Yuan et al. 2003). This quasar has higher X-ray to radio luminosity ratio $\left(\rho_{x r}>100\right)$ than any of its lower- $z$ counterparts (Cheung 2004). This appears consistent with the $(1+z)^{4}$ amplification in the energy density of Cosmic Microwave Background (CMB):

$$
\rho_{x r} \propto u_{\mathrm{CMB}} / u_{\mathrm{B}} \propto(1+z)^{4}(\delta / B)^{2},
$$

as expected under the IC/CMB model (e.g., Schwartz 2002).

Figure 2 compares the energy flux ratio $\rho_{x r}=\left[F_{\mathrm{x}}(0.5-7 \mathrm{keV})\right] /\left[\nu_{\mathrm{r}} f_{\mathrm{r}}\right]$ of the detected and marginally detected jets across redshift from our Chandra sample. We seek to establish whether or not the energy flux ratio varies with redshift. Figure 3 shows the posterior distribution from LIRA of the energy flux ratio for each detected and marginally detected source (blue corresponds to low, and red to high redshift). It is visually apparent that there is a difference in the distributions of the sources with higher redshifts $(z>3)$ and those with lower redshifts. In order to establish a statistical measure of the significance of this difference, we split the detected and marginally detected sources into two samples consisting of the 18 at low redshift $(z<3$; sample $L)$ and the 3 at high redshift $(z>3$; sample $H)$. We 
then use a hierarchical Gaussian model to examine whether low and high redshift quasars differ in terms of the mean and variance of their energy flux ratio. Appendix A describes a procedure for evaluating the posterior probability that the difference between the mean $\log _{10}$ energy flux ratio of the high and low redshift jets $\left(\mu_{H}-\mu_{L}\right)$ is greater than zero. Figure 4 shows the distribution of $\mu_{H}-\mu_{L}$ calculated from the posterior output. We find an empirical probability of $95 \%$ that $\mu_{H}-\mu_{L} \geq 0$, which is at best marginal evidence that the observed difference cannot be due to a statistical fluctuation. Though highly suggestive, because of the small number of sources represented in this paper, and given the disproportionate numbers of jets in the two samples, there is insufficient evidence to conclude that the mean $\log _{10}$ energy flux ratio differs between two groups of jets. More observations at $z>3$ are required in order to obtain more reliable results.

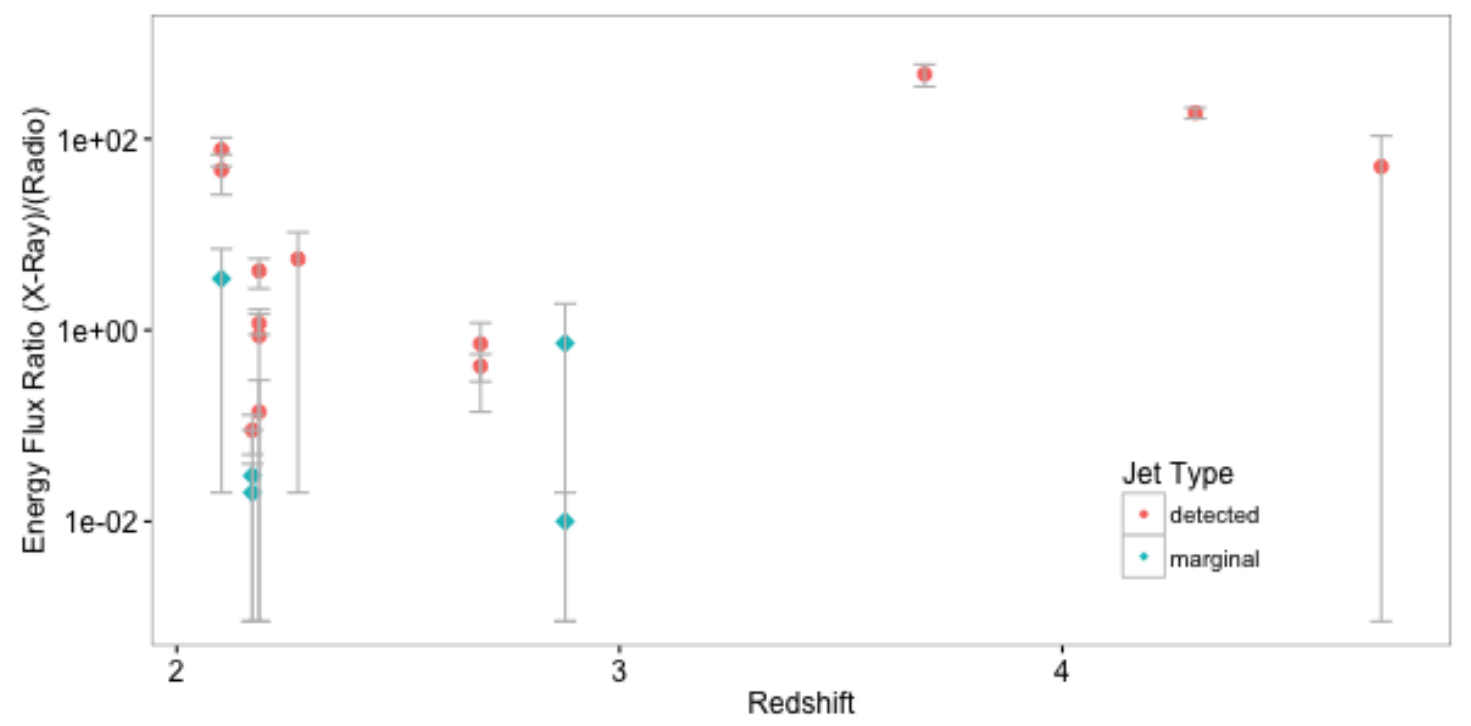

Fig. 2.- Ratio of X-ray to radio flux $\rho_{x r}$ vs redshift for the detected and marginally detected regions of interest of the jets. The circles are the energy flux ratios from jets detected in this study. The diamonds are estimated energy ratios from the marginally detected jets. The error bars form the $68 \%$ interval from the LIRA iterations.

The difference between the jets in two redshift groups is interesting because it can also indicate that radio-loud quasars at $z>3$ are different from low redshift ones. Volonteri et al. (2011) hypothesized that the jets at $z>3$ are systematically slower in comparison to the jets at $z<3$. If true, this could affect the energy flux ratio in the framework of the IC/CMB model, due to a strong dependence of this ratio on the jet Doppler factor. In this case, assuming that the comoving jet magnetic field is roughly the same at different redshifts, the observed increase in the energy flux ratio should be smaller than that expected from the 


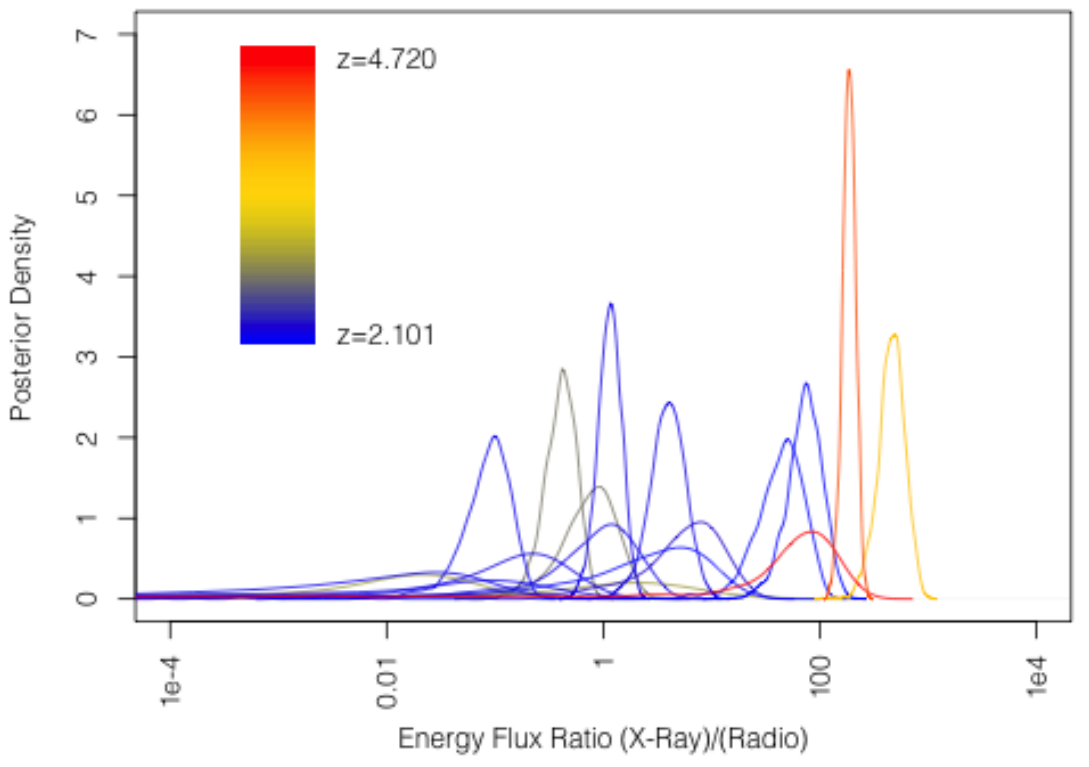

Fig. 3.- The posterior distribution of the ratio of X-ray to radio fluxes, $\rho_{x r}$, for each detected and marginally detected jet. The energy flux ratio is calculated at every iteration of LIRA. The color corresponds to redshift.

$(1+z)^{4}$ scaling. However, the jet magnetization may evolve with redshift (e.g. Singal et al. 2013) which complicates the redshift scaling even further. Our results indicate that the high redshift jets are different, but more observations are needed to study the origin of this difference.

\subsection{Quasars at High Redshift}

The X-ray emission of radio loud quasars observed with Chandra is unresolved and contained within $<1.5^{\prime \prime}$ circular regions. This emission could be due to a mixture of at least three components: a hot corona directly related to the accretion process, a parsec scale jet, and an unresolved portion of the kpc-scale outflow emitting X-rays via IC/CMB. We measured a standard range of photon indices for the assumed power law model for quasar core spectra and found them consistent with either process. However, we detected relatively low values of photon indices in a few quasars, including two at the highest redshift (see Table (4). Lower values of the photon index are predicted if the jet dominates the $\mathrm{X}$-ray 


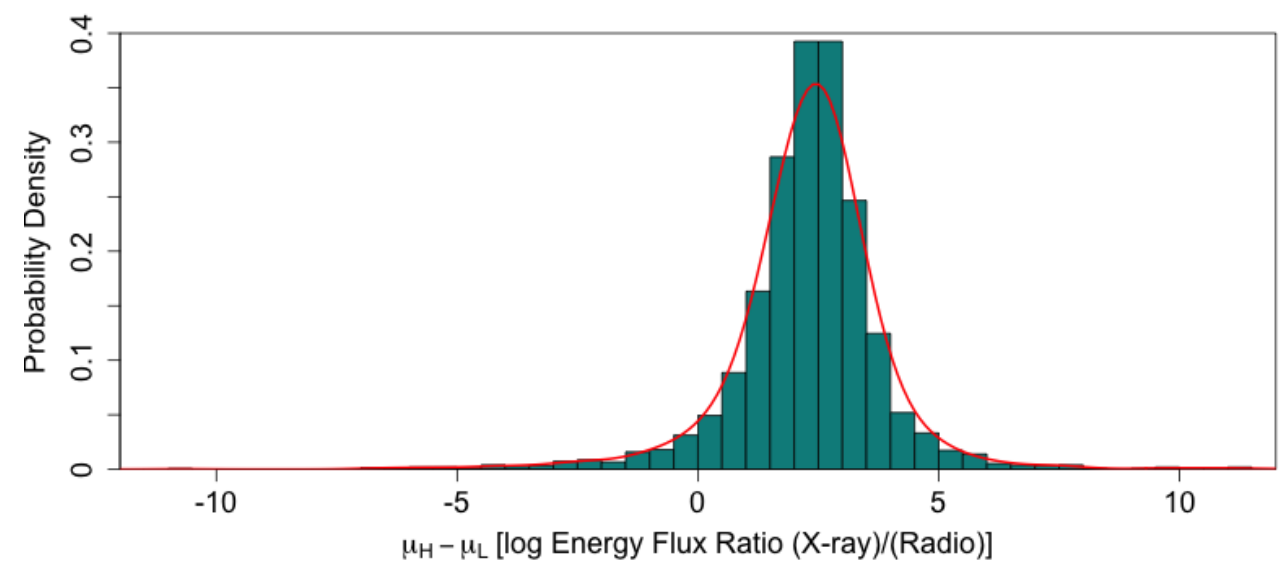

Fig. 4. - The difference in the mean X-ray to radio energy flux ratio between high- and medium-redshift quasars, $\mu_{H}-\mu_{L}$ at every LIRA iteration. $\mu_{L}$ and $\mu_{H}$ are the average $\log$ flux ratio across the lower-redshift $(2<z<3)$ and higher-redshift $(z>3)$ detected and marginally detected redshift jets.

emission. In this case the beamed jet would make the quasars to appear more luminous. We notice that such a trend is present in our small sample and five sources with $\Gamma_{X}<1.6$ are more luminous, with an average $2-10 \mathrm{keV}$ luminosity of $6.3 \pm 2.5 \times\left(10^{46} \mathrm{erg} \mathrm{s}^{-1}\right)$, than the other six with $\Gamma_{X}>1.6$ and the average luminosity of $7.9 \pm 2.5 \times\left(10^{45} \mathrm{erg} \mathrm{s}^{-1}\right)$. Formal correlation tests show that $\Gamma_{X}$ and $\log L_{X}$ are indeed correlated, with Pearson's correlation coefficient $\rho=0.61(p=0.047)$, and Kendall's $\tau=0.53(p=0.024)$. Such relation has been seen in analysis of large samples of radio-loud and radio-quiet quasars (Bechtold et al. 1994; Young et al. 2009; Lanzuisi et al. 2013). Bechtold et al. (1994) found a similar trend in a sample of radio loud quasars and argued that it could be caused by an increased absorption. On the other hand Young et al. (2009) did not find a significant correlation in a radio-loud subsample of quasars observed with XMM-Newton. Future studies of large number of radio loud quasars in X-ray and radio band are necessary for understanding the presence and origin of this correlation.

In the analysis of the Chandra images we assumed that the quasar emission is pointlike. However, we detect signatures of non-point-like emission in the two highest redshift $(z>4)$ quasars $(1428+422$ and $1508+5714)$ and in the one at $z=2.3(1834+612)$ (as evidenced by the fact that the core component $\mathrm{Q}$ is not fully accounted for in the baseline model, see Table 6 and Figures 8 13 14). This is unlikely to be due to uncertainties in the shape of the Chandra PSF, since the residual core emission is not present in the multi-scale 
components for other sources. We suggest that these are due to non-negligible contributions from unresolved kpc-scale jets emitting IC/CMB. The effect of this residual component on our analysis is conservative, i.e., the imperfect modeling of the core tends to increase the strength of the baseline model and systematically dampen the added multi-scale component. Thus, our results indicate that the unresolved X-ray cores of radio loud high redshift quasars can contain significant contributions from kpc-scale jet emission. This result is in agreement with studies based on the optical-to-X-ray luminosity ratio (Saez et al. 2011; Wu et al. 2013) and along the expectations from the IC/CMB model. Such a jet contribution can potentially bias population studies of quasars and needs to be taken into account in investigations of radio loud quasars at high redshift.

\section{Summary}

We have analyzed Chandra X-ray observations of a sample of eleven $z>2$ quasar radio jets including flat-spectrum and steep-spectrum radio sources. The quasars were selected based on the known radio jets. We use the Bayesian multi-scale image reconstruction method, LIRA, to obtain high-quality images outside the quasar core, and assess the significances of X-ray emission features coincident with jets. We detect X-ray counterparts to radio jets in 9 quasars, including in the highest redshift $\mathrm{X}$-ray and radio jet currently known (GB $1428+4217$ at $z=4.72$, Cheung et al.(2012)). In particular, 12 radio features are detected at high significance, and an additional 6 at marginal significance.

We find that the ratio of X-ray to radio energy flux may differ between jets at high $(z>3)$ and low $(z<3)$ redshift, in accordance with the IC/CMB mechanism for X-ray emission, or pointing to the intrinsic differences between low and high redshift quasars. However, this difference is subject to a large uncertainty due to small sample sizes, and more observations are needed in the high redshift regime to confirm this trend.

We remember Dan Harris' passion for understanding jets: "After all these years and all these conferences on jets, we still don?t know what jets are made of or how they work" (Harris 2015). The authors acknowledge Rita Sambruna's contribution as the PI of the Chandra X-ray Observatory program for a sub-sample of quasars analyzed in this work. A.S. thanks Francesco Massaro and Giulia Migliori for discussion. Doug Gobeille participated in the initial selection of the radio jets. This work was supported in part by the National Science Foundation REU and Department of Defense ASSURE programs under NSF Grant no. 1262851 and by the Smithsonian Institution. The methodological aspects of this project were conducted under the auspices of the CHASC International Astrostatstics Center. CHASC 
is supported by NSF grants DMS 1208791, DMS 1209232, DMS 1513546 and 1513492. DvD acknowledges support from a Wolfson Research Merit Award (WM110023) provided by the British Royal Society and from Marie-Curie Career Integration (FP7-PEOPLE-2012-CIG321865) and Marie-Skodowska-Curie RISE (H2020-MSCA-RISE-2015-691164) Grants both provided by the European Commission. This research was supported in part by NASA through contract NAS8-03060 (A.S., D.A.S., V.L.K.) to the Chandra X-ray Center. It was also supported by the Chandra Award number GO7-8114 to Brandeis University by the Chandra X-Ray Observatory Center, which is operated by the Smithsonian Astrophysical Observatory for and on behalf of NASA under contract NAS8-03060. L.S. was supported by Polish NSC grant DEC-2012/04/A/ST9/00083.

Facilities: CXO, VLA

\section{REFERENCES}

Adelman-McCarthy, J. K., Agüeros, M. A., Allam, S. S., et al. 2008, ApJS, 175, 297

Baker, J. C., Hunstead, R. W., Athreya, R. M., et al. 2002, ApJ, 568, 592

Bassett, L. C., Brandt, W. N., Schneider, D. P., et al. 2004, AJ, 128, 523

Beasley, A. J., Gordon, D., Peck, A. B., et al. 2002, ApJS, 141, 13

Bechtold, J., Elvis, M., Fiore, F., et al. 1994, AJ, 108, 759

Begelman, M. C., Blandford, R. D., \& Rees, M. J. 1984, Reviews of Modern Physics, 56, 255

Bridle, A. H., \& Perley, R. A. 1984, ARA\&A, 22, 319

Bridle, A. H., \& Greisen, E. W. 1994, AIPS Memo 87 (NRAO: Charlottesville)

Britzen, S., Vermeulen, R. C., Campbell, R. M., et al. 2008, A\&A, 484, 119

Celotti, A., Ghisellini, G., \& Chiaberge, M. 2001, MNRAS, 321, L1

Cheung, C. C. 2004, ApJ, 600, L23

Cheung, C. C., Wardle, J. F. C., \& Lee, N. P. 2005, in 22nd Texas Symposium on Relativistic Astrophysics, Ed. P. Chen et al., (Palo Alto: SLAC) 1613

Cheung, C. C., Stawarz, Ł., \& Siemiginowska, A. 2006, ApJ, 650, 679 
Cheung, C. C., Stawarz, Ł., Siemiginowska, A., et al. 2008, in "Extragalactic Jets: Theory and Observation from Radio to Gamma Ray," Eds. T. A. Rector \& D. S. De Young, ASP Conf.Ser. 386, 462

Cheung, C. C., Stawarz, Ł., Siemiginowska, A., et al. 2012, ApJ, 756, L20

Condon, J. J., Cotton, W. D., Greisen, E. W., et al. 1998, AJ, 115, 1693

Connors, A., \& van Dyk, D. A. 2007, Statistical Challenges in Modern Astronomy IV, 371, 101

Croton, D. J., Springel, V., White, S. D. M., et al. 2006, MNRAS, 365, 11

Davis, J. E. 2001, ApJ, 562, 575

De Young, D. 2006, Astron.Nachr., 327, 231

Dickey, J. M., \& Lockman, F. J. 1990, ARA\&A, 28, 215

Ellison, S. L., Yan, L., Hook, I. M., et al. 2001, A\&A, 379, 393

Esch, D. N., Connors, A., Karovska, M., \& van Dyk, D. A. 2004, ApJ, 610, 1213

Fey, A. L., Ma, C., Arias, E. F., et al. 2004, AJ, 127, 3587

Freeman, P., Doe, S., \& Siemiginowska, A. 2001, Proc. SPIE, 4477, 76

Fruscione, A., McDowell, J. C., Allen, G. E., et al. 2006, Proc. SPIE, 6270

Gobeille, D. B., Wardle, J. F. C., \& Cheung, C. C. 2014, arXiv:1406.4797

Gobeille, D. 2011, PhD thesis, Brandeis University

Gregory, P. C., \& Condon, J. J. 1991, ApJS, 75, 1011

Harris, D.E., \& Krawczynski, H. 2006, ARA\&A, 44, 463

Harris, D. E. 2015, Extragalactic Jets from Every Angle, 313, 199

Helmboldt, J. F., Taylor, G. B., Tremblay, S., et al. 2007, ApJ, 658, 203

Henstock, D. R., Browne, I. W. A., Wilkinson, P. N., \& McMahon, R. G. 1997, MNRAS, 290,380

Hook, I. M., \& McMahon, R. G. 1998, MNRAS, 294, L7 
Hook, I. M., McMahon, R. G., Patnaik, A. R., et al. 1995, MNRAS, 273, L63

Jester, S., Harris, D. E., Marshall, H. L., \& Meisenheimer, K. 2006, ApJ, 648, 900

Johnston, K. J., Fey, A. L., Zacharias, N., et al. 1995, AJ, 110, 880

Jorstad, S. G., Marscher, A. P., Mattox, J. R., et al. 2001, ApJS, 134, 181

Jorstad, S. G., \& Marscher, A. P. 2004, ApJ, 614, 615

Kalberla, P. M. W., Burton, W. B., Hartmann, D., et al. 2005, A\&A, 440, 775

Kapahi, V. K., Athreya, R. M., van Breugel, W., McCarthy, P. J., \& Subrahmanya, C. R. 1998, ApJS, 118, 275

Kashyap, V. L., van Dyk, D. A., Connors, A., et al. 2010, ApJ, 719, 900

Kataoka, J., \& Stawarz, Ł. 2005, ApJ, 622, 797

Kelly, B. C., Bechtold, J., Siemiginowska, A., Aldcroft, T., \& Sobolewska, M. 2007, ApJ, 657,116

Kühr, H. 1980, Ph.D. Thesis, Bonn

Lanzuisi, G., Civano, F., Elvis, M., et al. 2013, MNRAS, 431, 978

Lister, M. L., Cohen, M. H., Homan, D. C., et al. 2009, AJ, 138, 1874

Liu, F. K., \& Zhang, Y. H. 2002, A\&A, 381, 757

Loiseau, N., Reich, W., Wielebinski, R., Reich, P., \& Muench, W. 1988, A\&AS, 75, 67

Lonsdale, C. J., Barthel, P. D., \& Miley, G. K. 1993, ApJS, 87, 63

Lopez, L. A., Brandt, W. N., Vignali, C., et al. 2006, AJ, 131, 1914

Lynds, C. R., \& Wills, D. 1970, Nature, 226, 532

Lynds, R., \& Wills, D. 1972, ApJ, 172, 531

Ma, C., Arias, E. F., Eubanks, T. M., et al. 1998, AJ, 116, 516

Marshall, H. L., Gelbord, J. M., Schwartz, D. A., et al. 2011, ApJS, 193, 15

Massaro, F., Harris, D. E., Cheung, C. C. 2011, ApJS, 197, 24

Monet, D. G., Levine, S. E., Canzian, B., et al. 2003, AJ, 125, 984 
Murphy, D. W., Browne, I. W. A., \& Perley, R. A. 1993, MNRAS, 264, 298

Oke, J. B., \& Korycansky, D. G. 1982, ApJ, 255, 11

Paragi, Z., Frey, S., Gurvits, L. I., et al. 1999, A\&A, 344, 51

Punsly, B. 1995, AJ, 109, 1555

Rees, M. J. 1989, MNRAS, 239, 1P

Saez, C., Brandt, W. N., Shemmer, O., et al. 2011, ApJ, 738, 53

Sambruna, R. M., Urry, C. M., Tavecchio, F., et al. 2001, ApJ, 549, L161

Sambruna, R. M., Gambill, J. K., Maraschi, L., et al. 2004, ApJ, 608, 698

Schwartz, D. A. 2002, ApJ, 569, L23

Schwartz, D. A. 2014, Proc. SPIE, 9144, 91440S

Shepherd, M. C., Pearson, T. J., Taylor, G. B. 1994, BAAS, 26, 987

Si, S., van Dyk, D. A., von Hipple, T., Robinson, E., Webster, A., and Stenning, D. 2016, MNRAS, submitted

Siemiginowska, A., Smith, R. K., Aldcroft, T. L., et al. 2003, ApJ, 598, L15

Siemiginowska, A., Stawarz, Ł., Cheung, C. C., et al. 2007, ApJ, 657, 145

Simionescu, A., Stawarz, Ł., Ichinohe, Y., et al. 2016, ApJ, 816, L15

Singal, J., Petrosian, V., Stawarz, Ł., \& Lawrence, A. 2013, ApJ, 764, 43

Stark, A. A., Gammie, C. F., Wilson, R. W., et al. 1992, ApJS, 79, 77

Stein, N. M., van Dyk, D. A., Kashyap, V. L., Siemiginowska, A., 2015 ApJ, 813, 66; Paper I.

Strittmatter, P. A., Carswell, R. F., Gilbert, G., \& Burbidge, E. M. 1974, ApJ, 190, 509

Schwartz, D. A. 2014, Rev. Sci. Instr., 85, 061101

Tavecchio, F., Maraschi, L., Sambruna, R. M., \& Urry, C. M. 2000, ApJ, 544, L23

Taylor, G. B., Vermeulen, R. C., Readhead, A. C. S., et al. 1996, ApJS, 107, 37 
Vermeulen, R. C., Taylor, G. B., Readhead, A. C. S., \& Browne, I. W. A. 1996, AJ, 111, 1013

Veron, P., Veron-Cetty, M.-P., Djorgovski, S., et al. 1990, A\&AS, 86, 543

Villani, D., \& di Serego Alighieri, S. 1999, A\&AS, 135, 299

Volonteri, M., Haardt, F., Ghisellini, G., \& Della Ceca, R. 2011, MNRAS, 416, 216

Wasserstein, R.L., \& Lazar, N.A., 2016, The American Statistician, 70:2, 129-133, DOI: 10.1080/00031305.2016.1154108

Weisskopf, M. C., Brinkman, B., Canizares, C., et al. 2002, PASP, 114, 1

Worrall, D. M. 2009, A\&A Rev., 17, 1

Wu, J., Brandt, W. N., Miller, B. P., et al. 2013, ApJ, 763, 109

Xu, W., Readhead, A. C. S., Pearson, T. J., Polatidis, A. G., \& Wilkinson, P. N. 1995, ApJS, 99, 297

Young, M., Elvis, M., \& Risaliti, G. 2009, ApJS, 183, 17

Yuan, W., Fabian, A. C., Celotti, A., \& Jonker, P. G. 2003, MNRAS, 346, L7 
Table 1: Sample Summary of Basic Properties

\begin{tabular}{lccccccc}
\hline \hline Name & $\begin{array}{c}\text { R.A. } \\
(J 2000.0)\end{array}$ & $\begin{array}{c}\text { Decl. } \\
(J 2000.0)\end{array}$ & Ref. & $z$ & Ref. & $\begin{array}{c}D_{\mathrm{L}} \\
(\mathrm{Gpc})\end{array}$ & $\begin{array}{c}\text { Scale } \\
\left(\mathrm{kpc} /{ }^{\prime \prime}\right)\end{array}$ \\
\hline $0730+257$ & 073308.784 & +253625.06 & SDSS & 2.686 & O82 & 22.6 & 8.06 \\
$0805+046$ & 080757.539 & +043234.53 & F04 & 2.877 & L70 & 24.6 & 7.92 \\
$1311-270$ & 131347.401 & -271649.13 & USNO & 2.186 & B02 & 17.6 & 8.39 \\
$1318+113$ & 132118.838 & +110649.98 & SDSS & 2.171 & L72 & 17.4 & 8.39 \\
$1834+612$ & 183519.675 & +611940.01 & B02 & 2.274 & H97 & 18.4 & 8.34 \\
$0833+585$ & 083722.410 & +582501.85 & J95 & 2.101 & K80 & 16.7 & 8.43 \\
$1239+376$ & 124209.812 & +372005.69 & F04 & 3.818 & V96 & 34.5 & 7.22 \\
$1754+676$ & 175422.187 & +673736.36 & USNO & 3.60 & V99 & 32.2 & 7.38 \\
$1418-064$ & 142107.756 & -064356.36 & B02 & 3.689 & E01 & 33.2 & 7.31 \\
$1428+422$ & 143023.742 & +420436.49 & F04 & 4.72 & H98 & 44.5 & 6.59 \\
$1508+5714$ & 151002.922 & +570243.37 & M98 & 4.30 & H95 & 39.8 & 6.87 \\
\hline \hline
\end{tabular}

Notes - The quasar radio positions are taken from: B02 = Beasley et al. (2002), F04 = Fey et al. (2004), and J95 = Johnston et al. (1995), while the optical measurements are from SDSS DR6 (Adelman-McCarthy et al. 2008) and USNO B1.0 (Monet et al. 2003).

The redshifts $(z)$ are from: B02 = Baker et al. (2002), E01 = Ellison et al. (2001), H97 = Henstock et al. (1997), H98 = Hook \& McMahon (1998), K80 = Kühr (1980), L70 = Lynds \& Wills (1970), L72 = Lynds \& Wills (1972), O82 = Oke \& Korycansky (1982), V96 = Vermeulen et al. (1996), V99 = Villani \& di Serego Alighieri (1999), and M98 = Ma et al. (1998).

Luminosity distances $\left(D_{\mathrm{L}}\right)$ and scales assume the adopted cosmological parameters, $H_{0}=$ $71 \mathrm{~km} \mathrm{~s}^{-1} \mathrm{Mpc}^{-1}, \Omega_{\mathrm{M}}=0.27$, and $\Omega_{\Lambda}=0.73$. 
Table 2: VLA Data Summary

\begin{tabular}{|c|c|c|c|c|c|c|}
\hline Name & Program & Date & $\begin{array}{c}\text { Frequency } \\
(\mathrm{GHz})\end{array}$ & Array & $\begin{array}{l}\text { Exp. Time } \\
\text { (s) }\end{array}$ & $\begin{array}{c}\text { Beam*** } \\
\left({ }^{\prime \prime}\right)\end{array}$ \\
\hline $0730+257$ & AK353 & 1994 Mar 20 & 8.7 & A & 2440 & 0.35 \\
\hline $0805+046$ & AB560 & 1990 Mar 24 & 4.9 & A & 2420 & 0.4 \\
\hline $1311-270$ & AL119 & 1986 Apr 28 & 4.9 & A & 1210 & 0.7 \\
\hline $1318+113$ & AB322 & 1985 Mar 10 & 4.9 & A & 1590 & 0.25 \\
\hline $1834+612$ & AT165 & 1994 Sep 06 & 4.7 & B & 170 & 1.5 \\
\hline $0833+585$ & AL164 & 1987 Oct 09 & 4.9 & A & 5200 & 0.50 \\
\hline $1239+376$ & AT165 & 1994 Sep 06 & 4.7 & B & 170 & $4.2,1.4$ at $\mathrm{PA}=-64.5 \mathrm{deg}$ \\
\hline $1754+676$ & AC755 & 2004 Dec 04 & 1.4 & A & 1060 & $1.5,1.1$ at $\mathrm{PA}=19.7 \mathrm{deg}$ \\
\hline $1418-064$ & S 8723 & 2007 Jul 27 & 4.9 & A & 3570 & $0.66,0.38$ at $\mathrm{PA}=-20.7 \mathrm{deg}$ \\
\hline $1428+422^{*}$ & AC755 & 2004 Dec 06 & 1.4 & A & 2020 & $1.6,1.1$ at $\mathrm{PA}=52.7 \mathrm{deg}$ \\
\hline $1508+5714^{* *}$ & AM492 & 1995 Jul 14 & 1.4 & A & 300 & $1.5,1.0$ at $\mathrm{PA}=-4.4 \mathrm{deg}$ \\
\hline
\end{tabular}

Notes $-{ }^{*}$ The data for $1428+422$ were published in Cheung et al. (2012). ** The data for $1508+5714$ were published in Cheung (2004). ${ }^{* * *}$ The beam sizes are elliptical Gaussians with major axis ("), minor axis ("), at the position angles (PA) indicated, or circular Gaussians when a single dimension is indicated.

Table 3: Chandra X-ray Observation Summary

\begin{tabular}{lccc}
\hline \hline Name & ObsID & Date & $\begin{array}{c}\text { Net Exposure } \\
(\mathrm{ksec})\end{array}$ \\
\hline $0730+257$ & 10307 & 2009 Feb 12 & 20.1 \\
$0805+046$ & 10308 & 2009 Feb 20 & 19.2 \\
$1311-270$ & 10309 & 2009 Mar 19 & 18.3 \\
$1318+113$ & 10310 & 2009 Mar 05 & 18.3 \\
$1834+612$ & 10311 & 2009 May 07 & 17.2 \\
$0833+585$ & 7870 & 2007 Jan 12 & 3.8 \\
$1239+376$ & 7871 & 2007 Mar 10 & 4.7 \\
$1754+676$ & 7872 & 2007 May 25 & 6.5 \\
$1418-064$ & 7873 & 2007 Jun 04 & 3.3 \\
$1428+422^{*}$ & 7874 & 2007 Mar 26 & 10.6 \\
$1508+5714^{* *}$ & 2241 & 2001 Jun 10 & 88.9 \\
\hline \hline
\end{tabular}

Notes $-{ }^{*}$ The data for $1428+422$ were published in Cheung et al. (2012). ${ }^{* *}$ The data for $1508+5714$ were published in Siemiginowska et al. (2003). 


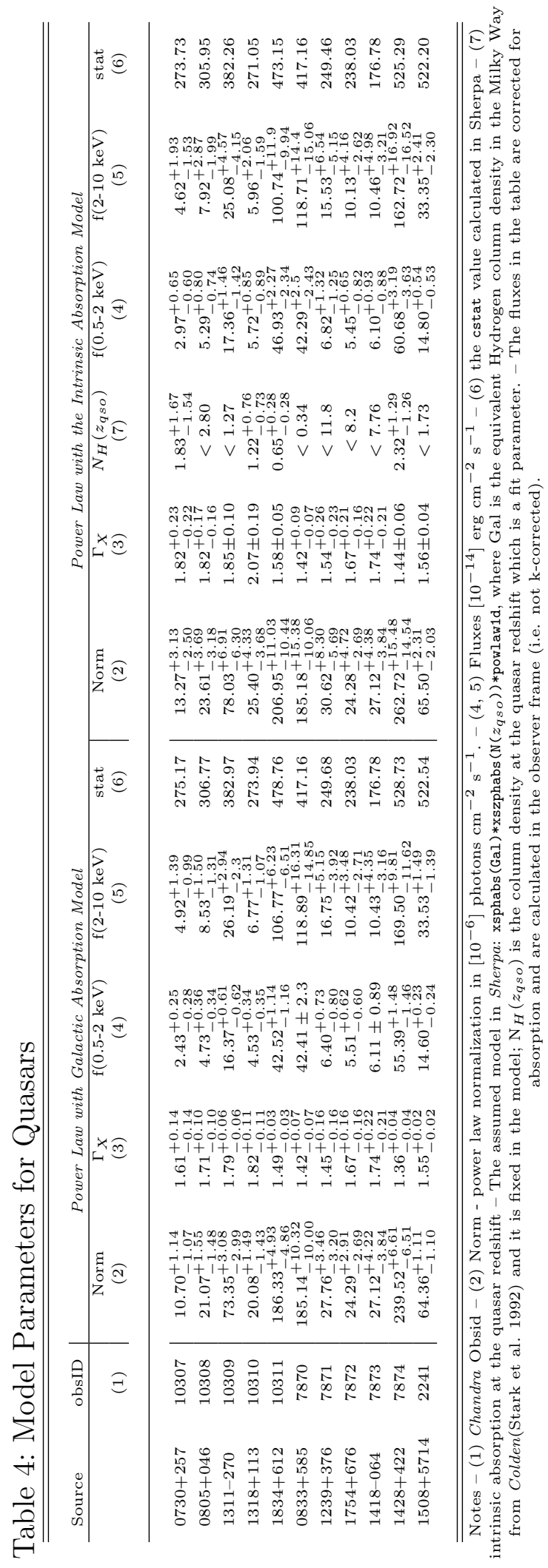


Table 5: Parameters for each region.

\begin{tabular}{|c|c|c|c|c|c|c|}
\hline ObsID & Region $^{a}$ & $\begin{array}{c}\text { Jet X-Ray Flux } \\
10^{-15}\left(\mathrm{erg} / \mathrm{s} / \mathrm{cm}^{2}\right)\end{array}$ & $\begin{array}{c}\text { Radio Flux } \\
10^{-15}\left(\mathrm{erg} / \mathrm{s} / \mathrm{cm}^{2}\right) \\
\end{array}$ & $\begin{array}{c}\text { p-value } \\
(\hat{u})\end{array}$ & $\begin{array}{c}\text { Signif. }^{c} \\
(\alpha=0.02)\end{array}$ & $\begin{array}{c}\text { Avg. } \\
\tau_{1} \\
\end{array}$ \\
\hline \multirow[t]{5}{*}{10307} & Q & 0.009 & 0.80 & 0.088 & & 0.82 \\
\hline & 1 & 0.003 & 0.76 & 0.792 & & 0.03 \\
\hline & 2 & 0.622 & 1.18 & 0.006 & Yes & 5.50 \\
\hline & 3 & 1.98 & 4.81 & 0.005 & Yes & 13.14 \\
\hline & $\mathrm{C}$ & & & 0.221 & & 6.39 \\
\hline \multirow[t]{5}{*}{10308} & $\mathrm{Q}$ & 0.023 & 0.61 & 0.132 & & 1.16 \\
\hline & 1 & 0.043 & 1.37 & 0.048 & & 0.39 \\
\hline & 2 & 0.140 & 16.00 & 0.018 & Marginal & 1.00 \\
\hline & 3 & 0.163 & 0.17 & 0.020 & Marginal & 0.77 \\
\hline & $\mathrm{C}$ & & & 0.075 & & 5.61 \\
\hline \multirow[t]{7}{*}{10309} & Q & 0.041 & 5.28 & 0.156 & & 0.64 \\
\hline & 1 & 0.004 & 0.43 & 1.000 & & 0.02 \\
\hline & 2 & 1.48 & 0.35 & 0.005 & Yes & 8.53 \\
\hline & 3 & 0.347 & 0.38 & 0.008 & Yes & 1.93 \\
\hline & 4 & 0.180 & 1.52 & 0.009 & Yes & 1.27 \\
\hline & 5 & 3.22 & 2.71 & 0.005 & Yes & 17.02 \\
\hline & $\mathrm{C}$ & & & 0.452 & & 5.05 \\
\hline \multirow[t]{6}{*}{10310} & Q & 0.046 & 0.36 & 0.238 & & 0.15 \\
\hline & 1 & 0.219 & 6.06 & 0.018 & Marginal & 1.24 \\
\hline & 2 & 0.022 & 4.52 & 0.432 & & 0.11 \\
\hline & 3 & 1.43 & 16.00 & 0.005 & Yes & 8.62 \\
\hline & 4 & 0.196 & 11.9 & 0.018 & Marginal & 1.09 \\
\hline & $\mathrm{C}$ & & & 0.036 & & 8.09 \\
\hline \multirow[t]{4}{*}{10311} & Q & 9.63 & 20.50 & 0.005 & Yes & 51.54 \\
\hline & 1 & 0.129 & 0.930 & 0.028 & & 0.67 \\
\hline & 2 & 0.486 & 0.0091 & 0.008 & Yes & 2.79 \\
\hline & $\mathrm{C}$ & & & 0.018 & Marginal & 13.10 \\
\hline \multirow[t]{5}{*}{7870} & Q & 0.383 & 35.00 & 0.068 & & 0.80 \\
\hline & 1 & 0.964 & 0.26 & 0.011 & Marginal & 1.10 \\
\hline & 2 & 8.05 & 0.10 & 0.005 & Yes & 9.82 \\
\hline & 3 & 6.88 & 0.15 & 0.005 & Yes & 8.54 \\
\hline & $\mathrm{C}$ & & & 0.057 & & 6.91 \\
\hline \multirow[t]{3}{*}{7871} & $\mathrm{Q}$ & 0.457 & 12.60 & 0.528 & & 0.81 \\
\hline & 1 & 0.059 & 0.06 & 0.950 & & 0.11 \\
\hline & $\mathrm{C}$ & & & 1.000 & & 2.41 \\
\hline \multirow[t]{3}{*}{7872} & $\mathrm{Q}$ & 0.143 & 0.75 & 0.731 & & 0.46 \\
\hline & 1 & 0.447 & 0.03 & 0.792 & & 0.90 \\
\hline & $\mathrm{C}$ & & & 0.559 & & 2.86 \\
\hline \multirow[t]{3}{*}{7873} & Q & 1.15 & 10.70 & 0.058 & & 2.24 \\
\hline & 1 & 15.6 & 0.03 & 0.005 & Yes & 17.19 \\
\hline & $\mathrm{C}$ & & & 0.528 & & 1.83 \\
\hline \multirow[t]{3}{*}{7874} & $\mathrm{Q}$ & 20.19 & 2.20 & 0.005 & Yes & 70.01 \\
\hline & 1 & 1.23 & 0.02 & 0.010 & Marginal & 4.26 \\
\hline & $\mathrm{C}$ & & & 1.000 & & 1.79 \\
\hline \multirow[t]{3}{*}{2241} & Q & 2.51 & 2.54 & 0.005 & Yes & 82.50 \\
\hline & 1 & 3.25 & 0.02 & 0.005 & Yes & 106.84 \\
\hline & $\mathrm{C}$ & & & 0.005 & Yes & 53.12 \\
\hline
\end{tabular}

${ }^{a}: \mathrm{Q}$ - excess emission in the quasar core region, excluding the quasar; 1 - 5 - numbering of identified jet regions based on radio data; $\mathrm{C}$ - a region complementary to the quasar and a jet (the entire image minus the quasar core and jet regions)

${ }^{b}$ : Jet X-Ray Flux is the Flux calculated using the multiscale counts, so the background and quasar are removed

${ }^{c}$ : - 'Yes' marks a significant detection $\alpha<0.01$; 'Marginal' marks a detection with $0.01<\alpha<0.02$ 
Table 6: X-ray Spectral Fits for selected Jets Regions

\begin{tabular}{lccccccc}
\hline \hline source & obsID & $\begin{array}{c}\text { region } \\
(1)\end{array}$ & $\begin{array}{c}\text { Norm } \\
(2)\end{array}$ & $\begin{array}{c}\Gamma_{X} \\
(3)\end{array}$ & $\begin{array}{c}\mathrm{f}(0.5-2 \mathrm{keV}) \\
\mathrm{f}(2-10 \mathrm{keV})\end{array}$ & $\begin{array}{c}\text { stat } \\
(5)\end{array}$ \\
\hline & & & & & & & \\
$0730+257$ & 10307 & 3 & $1.07_{-0.31}^{+0.39}$ & $1.93_{-0.48}^{+0.51}$ & $0.24_{-0.09}^{+0.08}$ & $0.31_{-0.17}^{+0.32}$ & 69.77 \\
& 10307 & 2 & $0.97_{-0.30}^{+0.37}$ & $1.67_{-0.47}^{+0.49}$ & $0.22_{-0.08}^{+0.07}$ & $0.38_{-0.22}^{+0.55}$ & 78.06 \\
$0805+046$ & 10308 & 2 & $0.51_{-0.20}^{+0.27}$ & $2.04_{-0.71}^{+0.77}$ & $0.12_{-0.05}^{+0.05}$ & $0.13_{-0.09}^{+0.29}$ & 46.57 \\
$1311-270$ & 10309 & 5 & $2.21_{-0.49}^{+0.57}$ & $2.82_{-0.48}^{+0.51}$ & $0.53_{-0.12}^{+0.12}$ & $0.17_{-0.08}^{+0.20}$ & 75.52 \\
& 10309 & 2 & $0.91_{-0.30}^{+0.38}$ & $1.48_{-0.48}^{+0.49}$ & $0.21_{-0.08}^{+0.08}$ & $0.51_{-0.3}^{+0.66}$ & 69.11 \\
$1318+113$ & 10310 & 2 & $0.91_{-0.27}^{+0.34}$ & $1.92_{-0.52}^{+0.55}$ & $0.20_{-0.08}^{+0.08}$ & $0.266_{-0.15}^{+0.37}$ & 61.89 \\
$1834+612$ & 10311 & 2 & $0.55_{-0.22}^{+0.31}$ & $1.05_{-0.54}^{+0.55}$ & $0.14_{-0.06}^{+0.05}$ & $0.58_{-0.38}^{+1.2}$ & 65.61 \\
$0833+585$ & 7870 & 2 & $3.09_{-1.12}^{+1.50}$ & $0.94_{-0.46}^{+0.47}$ & $0.76_{-0.33}^{+0.32}$ & $3.79_{-2.13}^{+6.35}$ & 76.34 \\
& 7870 & 3 & $5.02_{-1.49}^{+1.84}$ & $1.73_{-0.47}^{+0.49}$ & $1.13_{-0.39}^{+0.37}$ & $1.92_{-1.02}^{+2.6}$ & 77.39 \\
& 7870 & 1 & $0.46_{-0.34}^{+0.67}$ & $3.93_{-2.25}^{+3.10}$ & $0.16_{-0.05}^{+0.25}$ & $0.01_{-0.01}^{+0.04}$ & 8.27 \\
$1239+376$ & 7871 & 1 & $0.27_{-0.23}^{+0.48}$ & $4.73_{-2.59}^{+3.78}$ & $0.10_{-0.03}^{+0.20}$ & $<0.10$ & 7.79 \\
$1418-064$ & 7873 & 1 & $9.96_{-2.26}^{+2.64}$ & $1.64_{-0.34}^{+0.35}$ & $2.32_{-0.61}^{+0.5}$ & $4.33_{-1.82}^{+3.34}$ & 116.01 \\
$1428+422$ & 7874 & 1 & $2.39_{-0.59}^{+0.71}$ & $1.64_{-0.38}^{+0.39}$ & $0.56_{-0.13}^{+0.13}$ & $1.07_{-0.53}^{+0.93}$ & 95.17 \\
$1508+572$ & 2241 & 1 & $2.14_{-0.19}^{+0.20}$ & $1.83_{-0.14}^{+0.15}$ & $0.48_{-0.05}^{+0.04}$ & $0.71_{-0.17}^{+0.20}$ & 239.02
\end{tabular}

Notes - (1) Obsid and regions; (2) Norm [10-6] photons $\mathrm{cm}^{-2} \mathrm{~s}^{-1}$. - $(4,5)$ Fluxes $\left[10^{-14}\right]$ erg $\mathrm{cm}^{-2} \mathrm{~s}^{-1}$; - These are based on a simple absorbed power law fit with $\mathrm{NH}$ frozen at the Galactic values. The fluxes in the table are corrected for absorption and are calculated in the observer frame (i.e. not k-corrected). 


\section{A. Flux Ratio Test}

We are interested in determining whether the flux ratio, $f_{X} / f_{r}$, correlates with redshift and more specifically if there is a difference in the distribution of the flux ratios of sources with higher redshifts and those with lower redshifts. To investigate this, we split the detected and marginally detected jets into two redshift classes: low redshift $(z<3)$ and high redshift $(z \geq 3)$ and denote the $\log _{10}$ flux ratio for the jet in region $i$ by $R_{i}=\log _{10}\left(f_{X}^{i} / f_{r}^{i}\right)$, for $i=1, . .18$. Of these, the first 15 ratios correspond to regions of low redshift sources and the remaining three to regions with high redshift sources. We postulate that

$$
\begin{aligned}
& R_{i} \stackrel{\text { indep }}{\sim} N\left(\mu_{L}, \sigma_{L}\right) \text { for low } z \text {, i.e., for } i=1, \ldots, 15 \\
& R_{i} \stackrel{\text { indep }}{\sim} N\left(\mu_{H}, \sigma_{H}\right) \text { for high } z \text {, i.e., for } i=16, \ldots, 18
\end{aligned}
$$

where $N(\mu, \sigma)$ denotes a normal distribution with mean $\mu$ and standard deviation $\sigma$. We independently assume non-informative priors for the parameters in Equation A1, i.e., $p\left(\mu_{L}, \sigma_{L}^{2}\right) \propto$ $1 / \sigma_{L}$ and $p\left(\mu_{H}, \sigma_{H}^{2}\right) \propto 1 / \sigma_{H}$. The distributions in Equation A1 can be viewed as a hierarchical prior on $R=\left(R_{1}, \ldots, R_{18}\right)$, hierarchical because it is specified in terms of parameters that are themselves fit to the data. We denote this hierarchical prior distribution by $p\left(R \mid \mu_{H}, \mu_{L}, \sigma_{H}, \sigma_{L}\right)$.

We are interested in whether or not the difference $\mu_{H}-\mu_{L}$ is greater than zero, which can be estimated via a Monte Carlo sample from the posterior distribution,

$$
p\left(\mu_{H}, \mu_{L}, \sigma_{H}, \sigma_{L}, R \mid y_{\mathrm{obs}}\right)
$$

where, using the notation of Paper I, $y_{\text {obs }}$ represents the observed data. A Monte Carlo sample from Equation A2 can be obtained by iteratively sampling $p\left(R \mid \mu_{H}, \mu_{L}, \sigma_{H}, \sigma_{L}, y_{\text {obs }}\right)$ and $p\left(\mu_{H}, \mu_{L}, \sigma_{H}, \sigma_{L} \mid R\right)$, i.e., via a Gibbs sampler. The second step is straightforward in that it involves a standard Bayesian fitting of the Gaussian distributions in Equation A1. We now turn our attention to using LIRA to obtain a sample from $p\left(R \mid \mu_{H}, \mu_{L}, \sigma_{H}, \sigma_{L}, y_{\text {obs }}\right)$.

Each $R_{i}$ is a deterministic function of $\tau_{1}$, the expected count from the added component under the LIRA model in the region corresponding to $R_{i}$; again we are using the notation of Paper I. Specifically,

$$
R_{i}=\log _{10}\left(\frac{f_{X}^{i}}{f_{r}^{i}}\right)=\log _{10}\left(\frac{\tau_{1} c}{E_{i} f_{r}^{i}}\right)
$$

where $E_{i}$ is the average exposure in region $i$ and $c$ is a flux to photon counts conversion factor. Thus, we can apply the transformation in Equation A3 to the Monte Carlo sample of $\tau_{1}$ generated by LIRA to obtain a sample of $R_{i}$. 
The prior distribution used for $\tau_{1}$ in LIRA, however, does not correspond to the hierarchical one given in Equation A1, but rather a gamma distribution? $\tau_{1} \sim \pi_{i} \operatorname{GAMMA}(a=$ $1, b=20$ ), where $\pi_{i}$ is the proportion of the image pixels that are in region $i$. Applying the transformation in Equation A3 to this prior distribution yields the prior implicitly assumed by LIRA for $R_{i}$,

$$
R_{i} \stackrel{\text { indep }}{\sim} \log _{e} \operatorname{GAMMA}\left(a=1, b=\frac{c \pi_{i}}{20 E_{i} f_{r}^{i}}\right) .
$$

We denote this prior distribution by $p_{\text {LIRA }}(R)=\prod_{i=1}^{18} p_{\text {LIRA }}\left(R_{i}\right)$. The difference between $p_{\text {LIRA }}(R)$ and $p(R)$ means that LIRA produce a Monte Carlo sample from

$$
p_{\mathrm{LIRA}}\left(R \mid y_{\mathrm{obs}}\right) \propto \frac{p\left(R \mid \mu_{H}, \mu_{L}, \sigma_{H}, \sigma_{L}, y_{\mathrm{obs}}\right) p_{\mathrm{LIRA}}(R)}{p\left(R \mid \mu_{H}, \mu_{L}, \sigma_{H}, \sigma_{L}\right)}
$$

To derive this expression, note that more precisely, LIRA provides a Monte Carlo sample from the joint posterior distribution, $p\left(\theta, R \mid y_{\text {obs }}\right)$, where $\theta$ represents a number of other unknown parameters that are not pertinent to the current discussion. Thus,

$$
\begin{aligned}
p_{\text {LIRA }}\left(R \mid y_{\text {obs }}\right) & =\int p_{\text {LIRA }}\left(\theta, R \mid y_{\text {obs }}\right) \mathrm{d} \theta \\
& =\int \frac{p\left(y_{\text {obs }}, \mid \theta, R\right) p(\theta \mid R) p_{\text {LIRA }}(R)}{p_{\text {LIRA }}\left(y_{\text {obs }}\right)} \mathrm{d} \theta \\
& =\frac{p_{\text {LIRA }}(R)}{p_{\text {LIRA }}\left(y_{\text {obs }}\right)} \int p\left(y_{\text {obs }}, \mid \theta, R\right) p(\theta \mid R) \mathrm{d} \theta
\end{aligned}
$$

where

$$
p_{\text {LIRA }}\left(y_{\text {obs }}\right)=\int p\left(y_{\text {obs }}, \mid \theta, R\right) p(\theta \mid R) p_{\text {LIRA }}(R) \mathrm{d} \theta \mathrm{d} R
$$

Because $y_{\mathrm{obs}}$ and $\left(\mu_{H}, \mu_{L}, \sigma_{H}, \sigma_{L}\right)$ are conditionally independent given $R$, we can write our target posterior distribution

$$
\begin{aligned}
p\left(R \mid \mu_{H}, \mu_{L}, \sigma_{H}, \sigma_{L}, y_{\mathrm{obs}}\right) & =\int p\left(\theta \mid R, y_{\mathrm{obs}}\right) p\left(R \mid \mu_{H}, \mu_{L}, \sigma_{H}, \sigma_{L}, y_{\mathrm{obs}}\right) \mathrm{d} \theta \\
& =\int \frac{p\left(y_{\mathrm{obs}}, \mid \theta, R\right) p(\theta \mid R) p\left(R \mid \mu_{H}, \mu_{L}, \sigma_{H}, \sigma_{L}\right)}{p\left(y_{\mathrm{obs}}\right)} \mathrm{d} \theta \\
& =\frac{p\left(R \mid \mu_{H}, \mu_{L}, \sigma_{H}, \sigma_{L}\right),}{p\left(y_{\mathrm{obs}}\right)} \int p\left(y_{\mathrm{obs}}, \mid \theta, R\right) p(\theta \mid R) \mathrm{d} \theta
\end{aligned}
$$

\footnotetext{
${ }^{7} \mathrm{~A}$ Gamma distribution with shape parameter $a$ and rate parameter $b$ has probability density function $\operatorname{pdf}(x)=\frac{b^{a}}{\Gamma(a)} x^{a-1} e^{-b x}$, mean $a / b$, and standard deviation $\sqrt{a} / b$.
} 
where

$$
p\left(y_{\mathrm{obs}}\right)=\int p\left(y_{\mathrm{obs}}, \mid \theta, R\right) p(\theta \mid R) p\left(R \mid \mu_{H}, \mu_{L}, \sigma_{H}, \sigma_{L}\right) \mathrm{d} \theta \mathrm{d} R .
$$

Finally combining Equations A6 and A7, we have

$$
\begin{aligned}
p_{\text {LIRA }}\left(R \mid y_{\text {obs }}\right) & =\frac{p\left(R \mid \mu_{H}, \mu_{L}, \sigma_{H}, \sigma_{L}, y_{\text {obs }}\right) p\left(y_{\text {obs }}\right) p_{\text {LIRA }}(R)}{p_{\text {LIRA }}\left(y_{\text {obs }}\right) p\left(R \mid \mu_{H}, \mu_{L}, \sigma_{H}, \sigma_{L}\right)} \\
& \propto \frac{p\left(R \mid \mu_{H}, \mu_{L}, \sigma_{H}, \sigma_{L}, y_{\text {obs }}\right) p_{\text {LIRA }}(R)}{p\left(R \mid \mu_{H}, \mu_{L}, \sigma_{H}, \sigma_{L}\right)} .
\end{aligned}
$$

Our strategy is to use the LIRA posterior as a proposal rule in a Metropolis Hastings sampler to obtain a sample from $p\left(R \mid \mu_{H}, \mu_{L}, \sigma_{H}, \sigma_{L}, y_{\text {obs }}\right)$. This can be accomplished using the following algorithm. A similar strategy is employed by Si et al. (2016) when they use Monte Carlo samples from the posterior distribution of the age of a number of white dwarfs obtained from separate fits as a Metropolis proposal rule for fitting a hierarchical model for all of their ages.

\section{Sampling Algorithm:}

Step 1: Run LIRA on each of the 18 regions. For region $i$, transform the sampled values of $\tau_{1}$ to $R_{i}$ using Equation A3. Concatenate the Monte Carlo sample of $R_{1}, \ldots, R_{18}$ to obtain the LIRA Monte Carlo sample of $R$. (Appropriate burn in and convergence checks should be implemented per standard practice with Markov chain Monte Carlo.)

Step 2: Set $R^{(0)}$ to a randomly selected value from the LIRA Monte Carlo sample of $R$. Fit the model in Equation A1 to $R^{(0)}$ using standard Bayesian techniques to obtain $\mu_{L}^{(0)}, \mu_{H}^{(0)}, \sigma_{L}^{(0)}$, and $\sigma_{H}^{(0)}$.

Step 3: For $t=1, \ldots, T$

Step 3a: Randomly select a proposal, $R_{\text {prop }}$, from the LIRA Monte Carlo sample of $R$.

Step 3b: Compute the 18 Metropolis Hastings acceptance probabilities,

$$
\begin{aligned}
r_{i} & =\frac{p\left(R_{i}^{\text {prop }} \mid\left(\mu_{L}, \mu_{H}, \sigma_{L}, \sigma_{H}\right)^{(t-1)}, y_{\mathrm{obs}}\right) p_{\mathrm{LIRA}}\left(R_{i}^{(t-1)} \mid y_{\mathrm{obs}}\right)}{p_{\mathrm{LIRA}}\left(R_{i}^{\mathrm{prop}} \mid y_{\mathrm{obs}}\right) p\left(R_{i}^{(t-1)} \mid\left(\mu_{L}, \mu_{H}, \sigma_{L}, \sigma_{H}\right)^{(t-1)}, y_{\mathrm{obs}}\right)} \\
& =\frac{p\left(R_{i}^{\text {prop }} \mid\left(\mu_{L}, \mu_{H}, \sigma_{L}, \sigma_{H}\right)^{(t-1)}\right) p_{\mathrm{LIRA}}\left(R_{i}^{(t-1)}\right)}{p_{\mathrm{LIRA}}\left(R_{i}^{\text {prop }}\right) p\left(R_{i}^{(t-1)} \mid\left(\mu_{L}, \mu_{H}, \sigma_{L}, \sigma_{H}\right)^{(t-1)}\right)} \\
& =\frac{N\left(R_{i}^{\text {prop }} ; \mu^{(t-1)}, \sigma^{(t-1)}\right)}{\log _{e} \Gamma\left(R_{i}^{\text {prop }} ; a=1, b=b_{i}\right)} \frac{\log _{10} \Gamma\left(R_{i}^{(t-1)} ; a=1, b=b_{i}\right)}{N\left(R_{i}^{(t-1)} ; \mu^{(t-1)}, \sigma^{(t-1)}\right)},
\end{aligned}
$$

where $N(R ; \mu, \sigma)$ is the probability density function of a normal variable with mean $\mu$ and variance $\sigma$ evaluated at $R, \log _{e} \Gamma(R ; a, b)$ is the probability density function of the 
$\log _{e}$ a gamma variable with parameters $a$ and $b$ evaluated at $R, b_{i}=c \pi_{i} /\left(20 E_{i} f_{r}^{i}\right)$, $\mu^{(t-1)}=\mu_{L}^{(t-1)}$ and $\sigma^{(t-1)}=\sigma_{L}^{(t-1)}$ for $i=1, \ldots, 15$, and $\mu^{(t-1)}=\mu_{H}^{(t-1)}$ and $\sigma^{(t-1)}=$ $\sigma_{H}^{(t-1)}$ for $i=15, \ldots, 18$

Step 3c: For $i=1, \ldots, 18$, set

$$
R_{i}^{(t)}= \begin{cases}R_{i}^{\text {prop }} & \text { with probability } \min \left(1, r_{i}\right) \\ R_{i}^{(t-1)} & \text { otherwise. }\end{cases}
$$

Step 3d: Sample $\mu_{L}^{(t)}, \mu_{H}^{(t)}, \sigma_{L}^{(t)}$, and $\sigma_{H}^{(t)}$ from $\left(\mu_{L}, \mu_{H}, \sigma_{L}, \sigma_{H} \mid R^{(t)}\right)$ using standard Bayesian methods.

Step 4: A Monte Carlo estimate of the posterior probability, $\operatorname{Pr}\left(\mu_{H}-\mu_{L}>0 \mid R\right)$ is given by the proportion of the Monte Carlo sample for which $\mu_{H}^{(t)}-\mu_{L}^{(t)}>0$.

Because this sampling algorithm in a Markov chain Monte Carlo sampler, appropriate burn in and convergence checks should be implemented.

\section{B. Additional Quasar Jet Observations and Analyses Figures}

As discussed in Section 3, we analyze a total of eleven quasar jets using LIRA. Here we display the radio and X-ray data as well as the results of the analyses for the remaining ten observations in the same form as Quasar 0730+257 (ObsID 10307) in Figure 1 . 
(a)

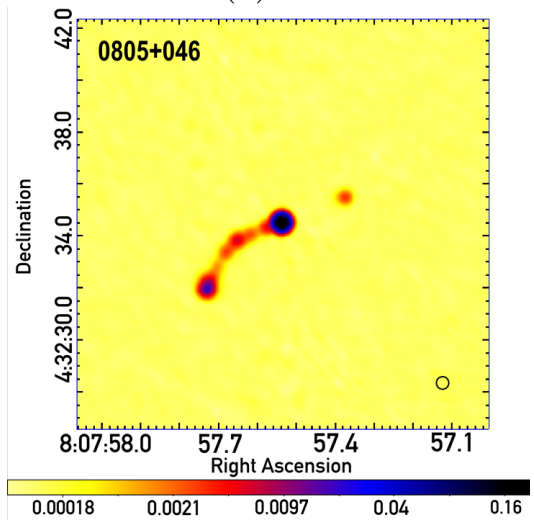

(b)

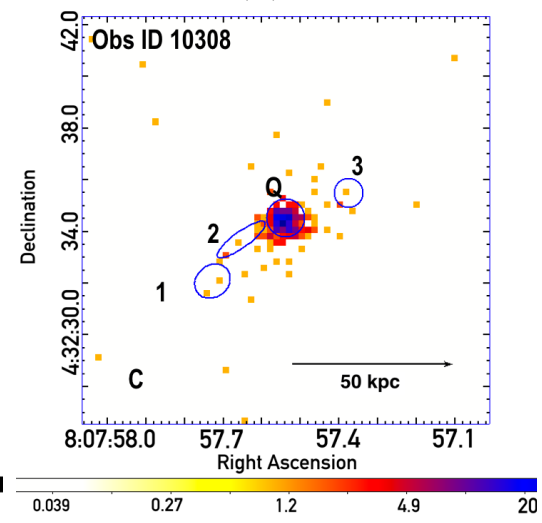

(d)
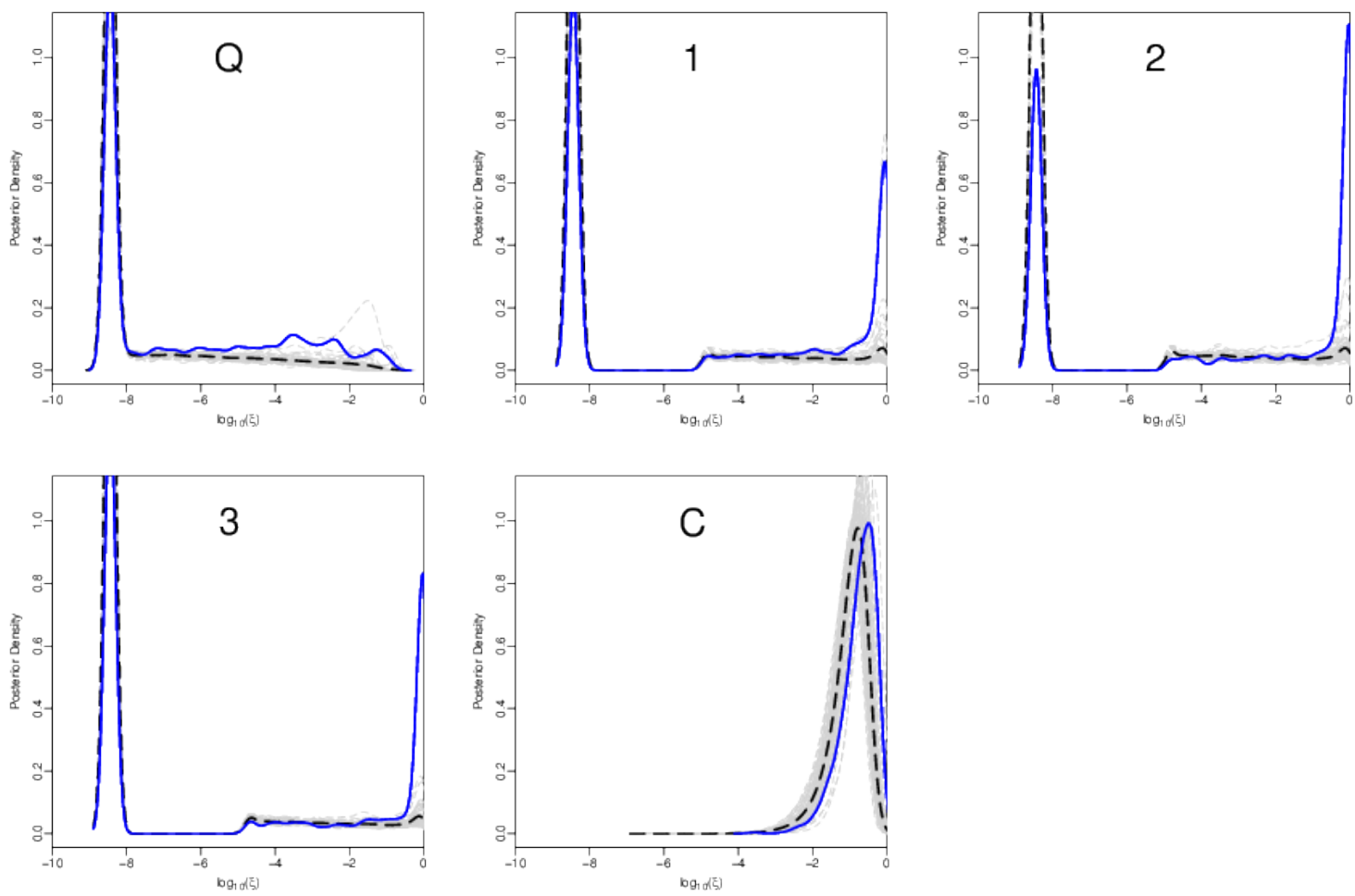

(c)

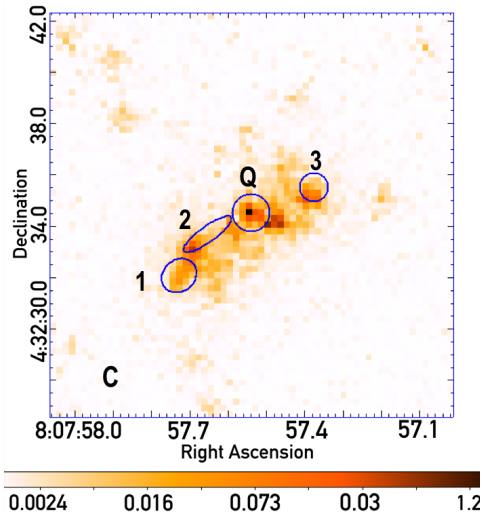

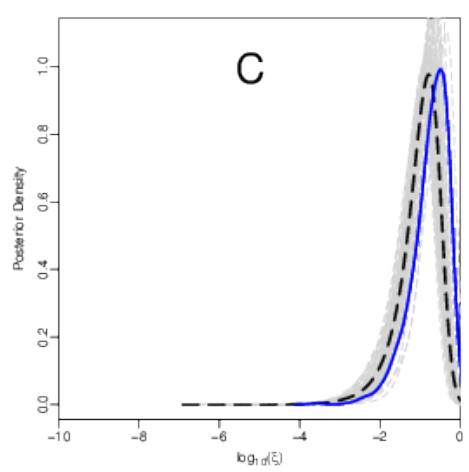

Fig. 5.- As in Figure 1,for quasar 0805+046 (ObsID 10308). 
(a)
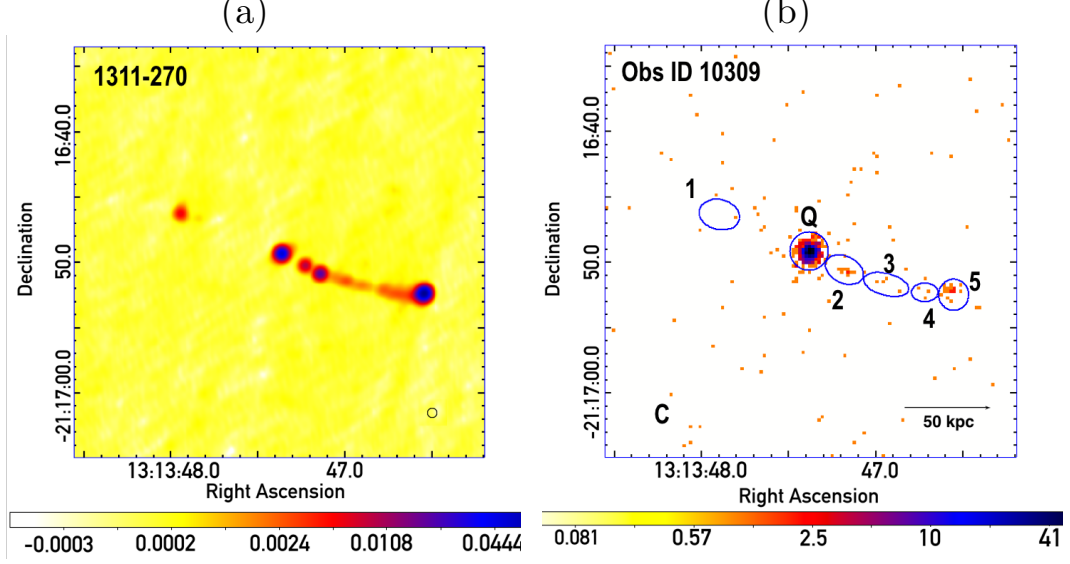

(d)
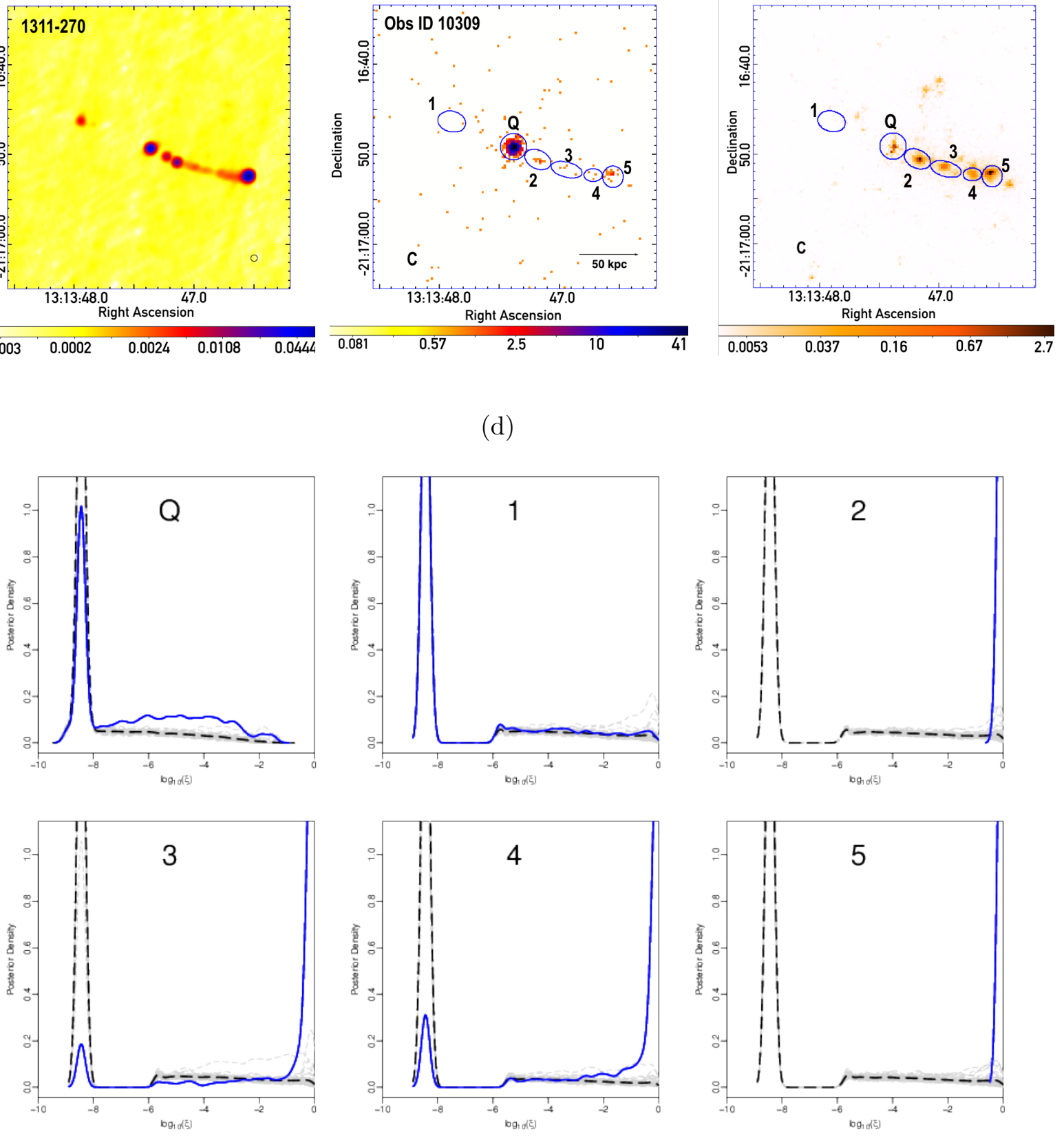

\section{)}

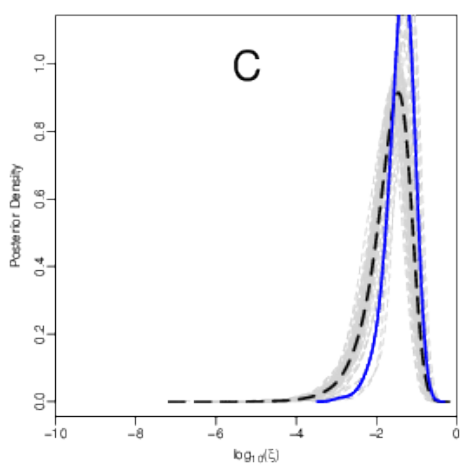


(a)
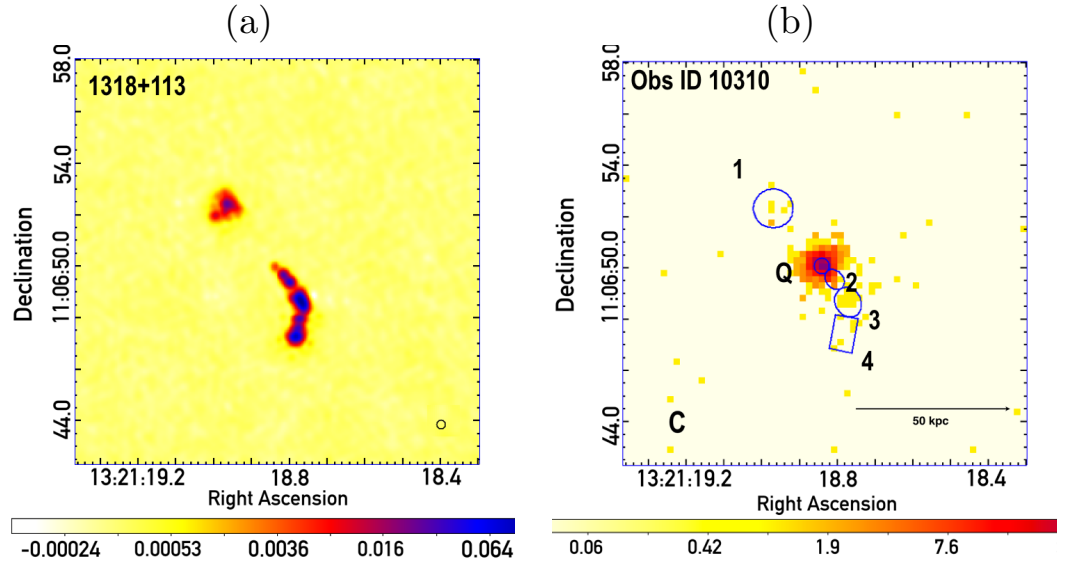

(d)
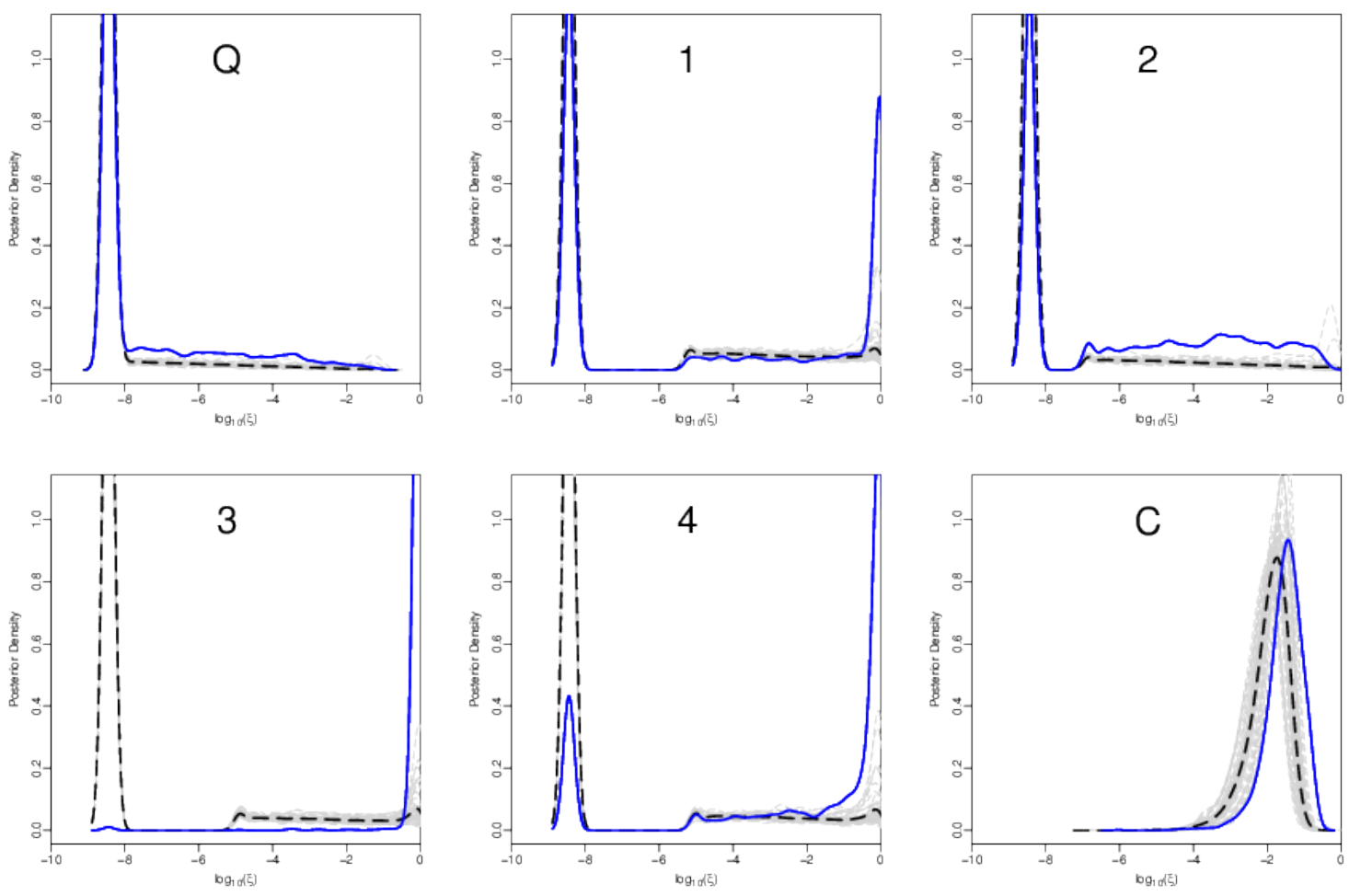

Fig. 7.- As in Figure 1, for quasar 1318+113 (ObsID 10310). 
(a)
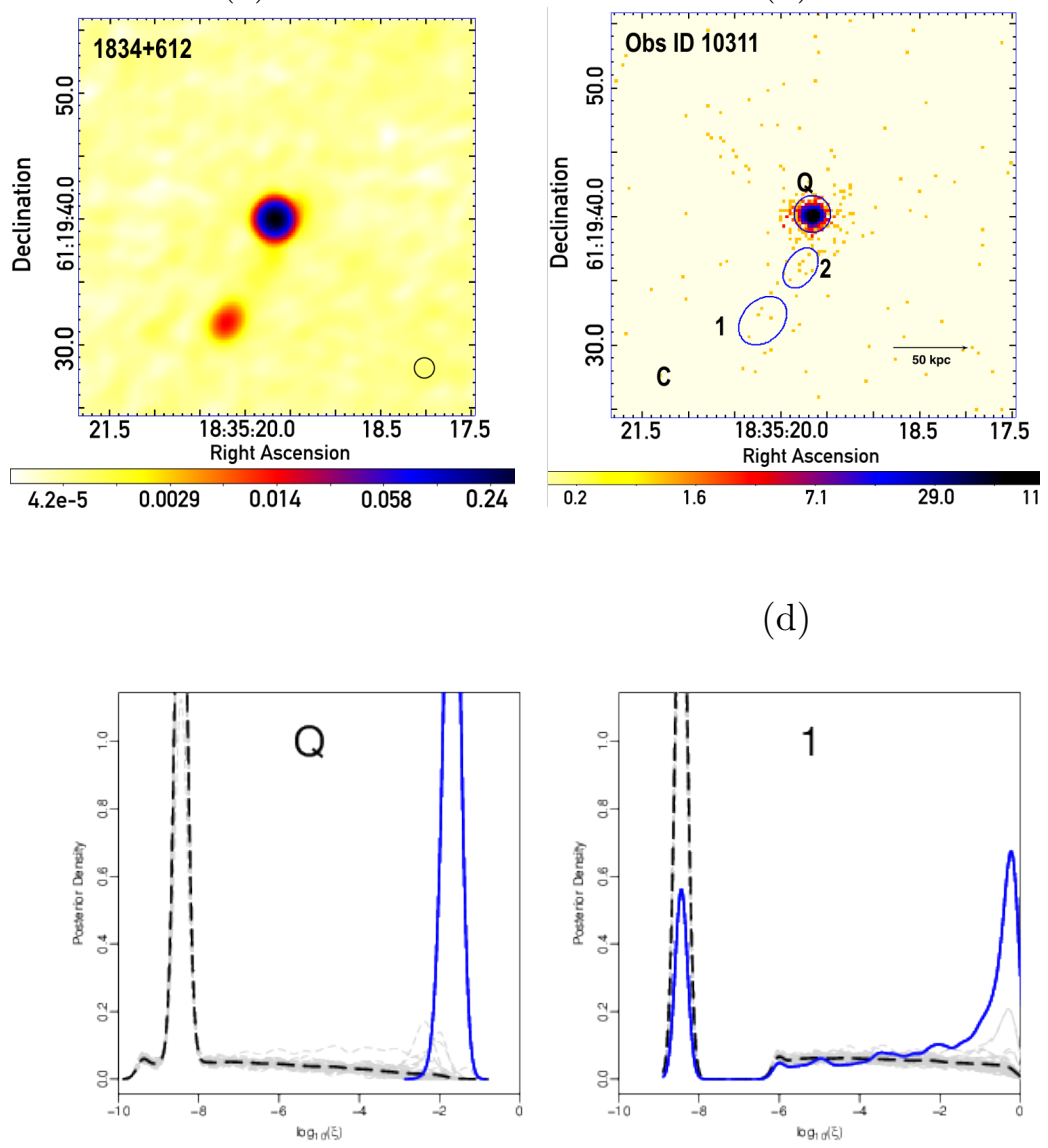

(d)

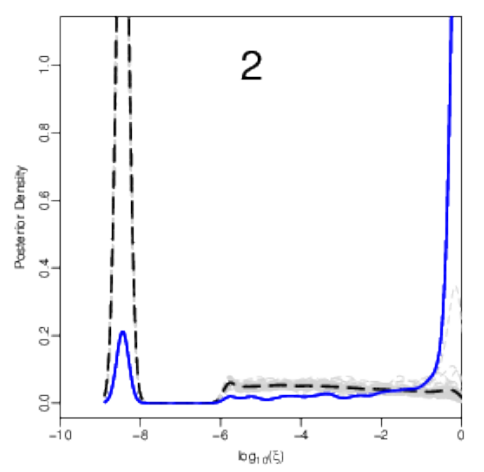

(c)

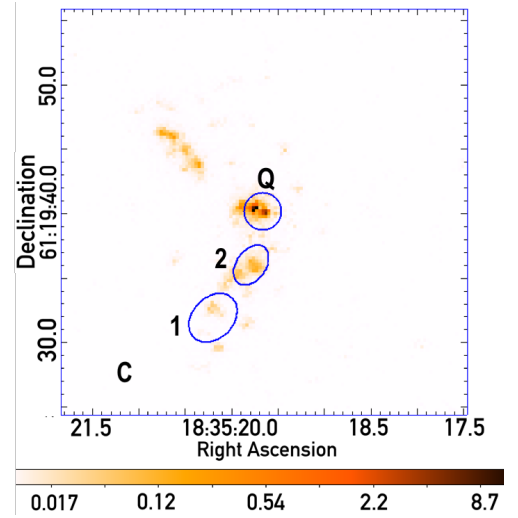

8.7

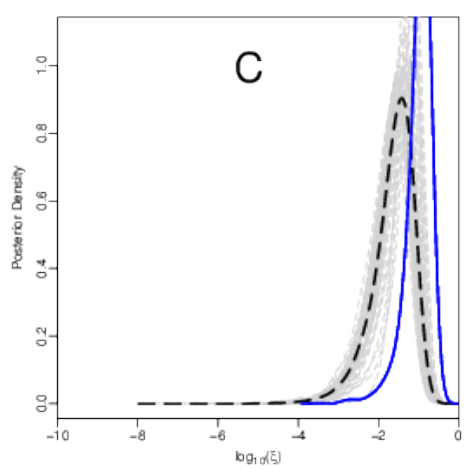


(a)
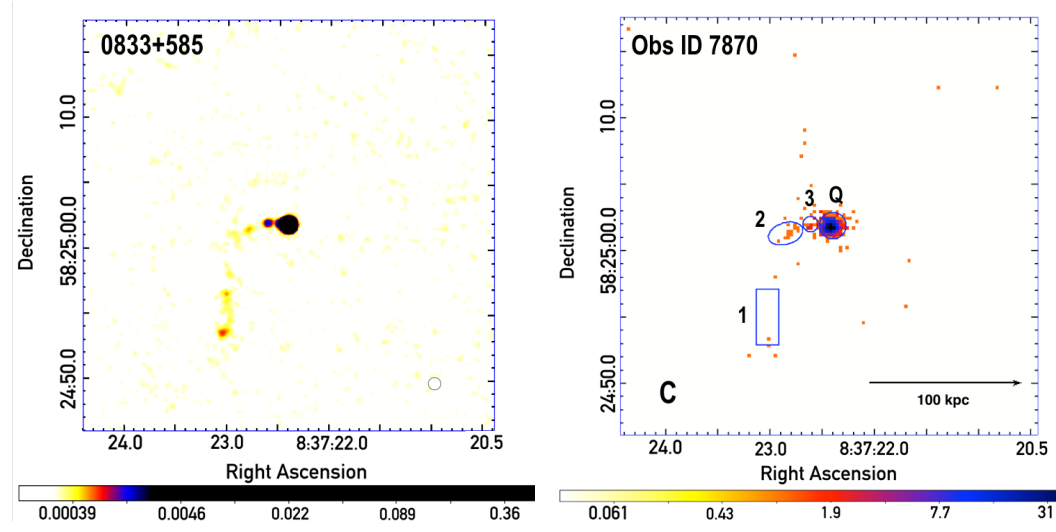

(d)
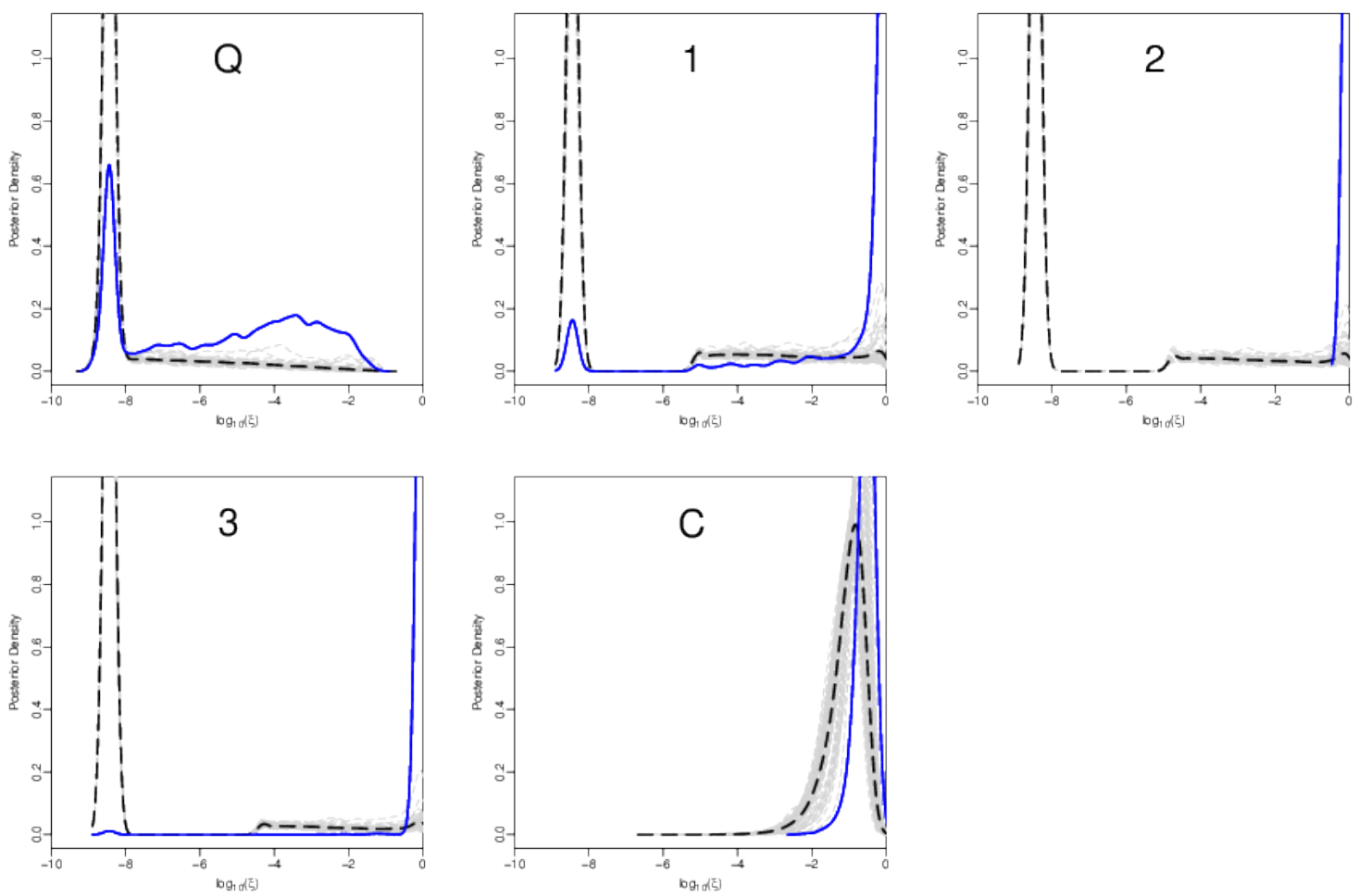

(c)

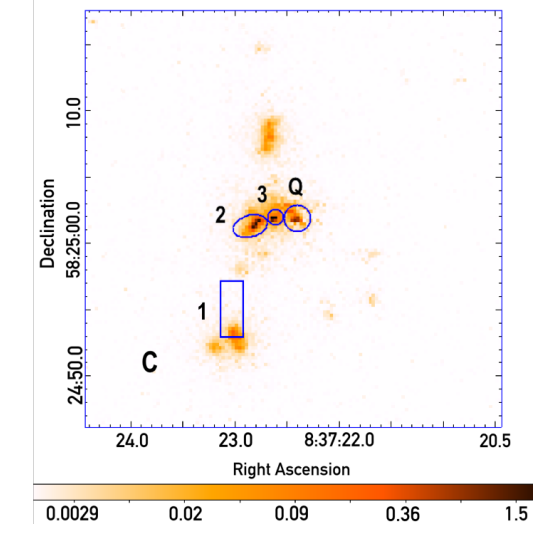


(a)
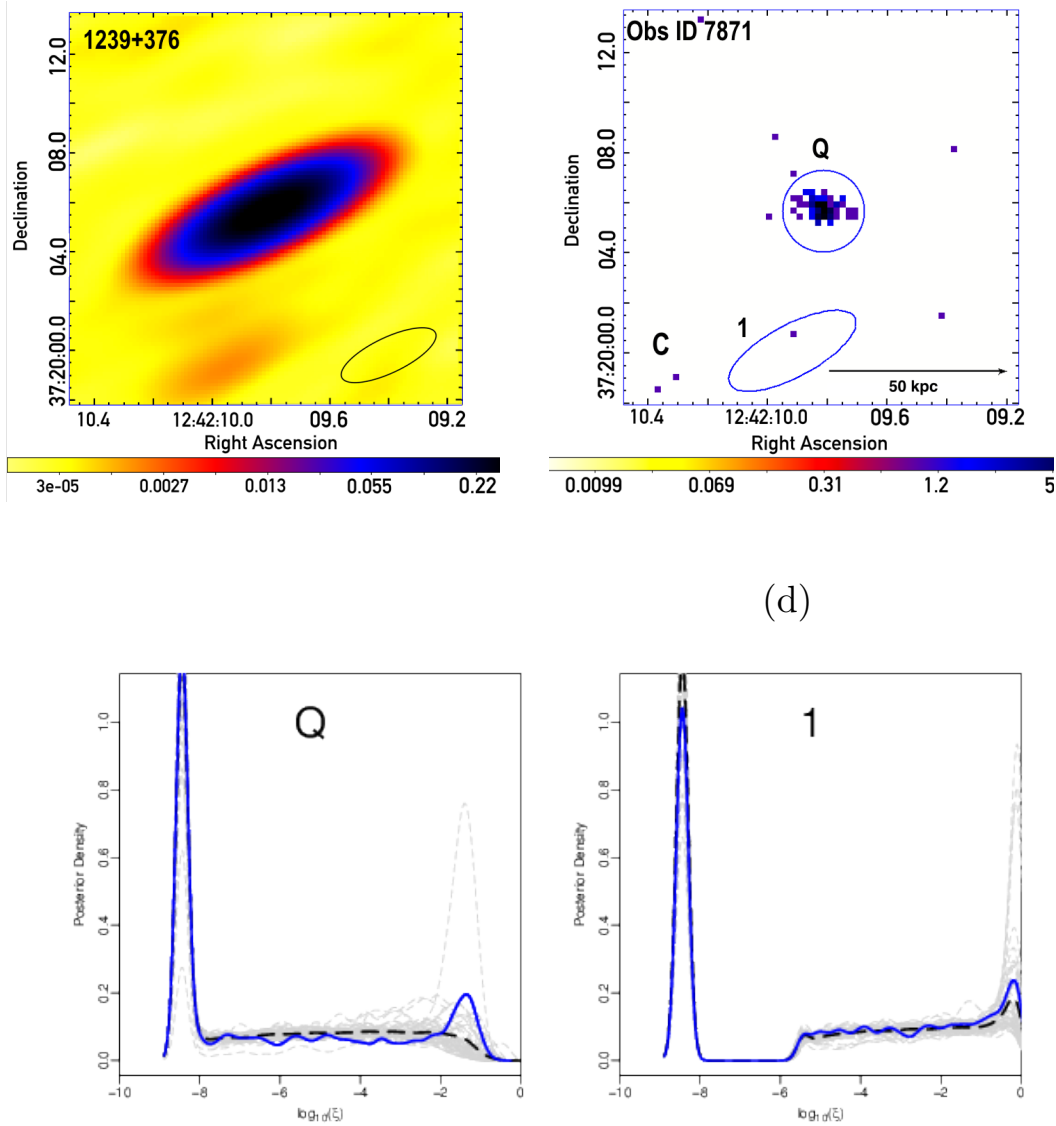

(b)

(d)

(c)

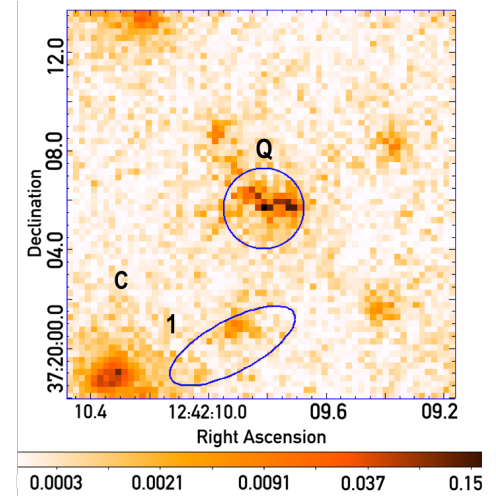

)
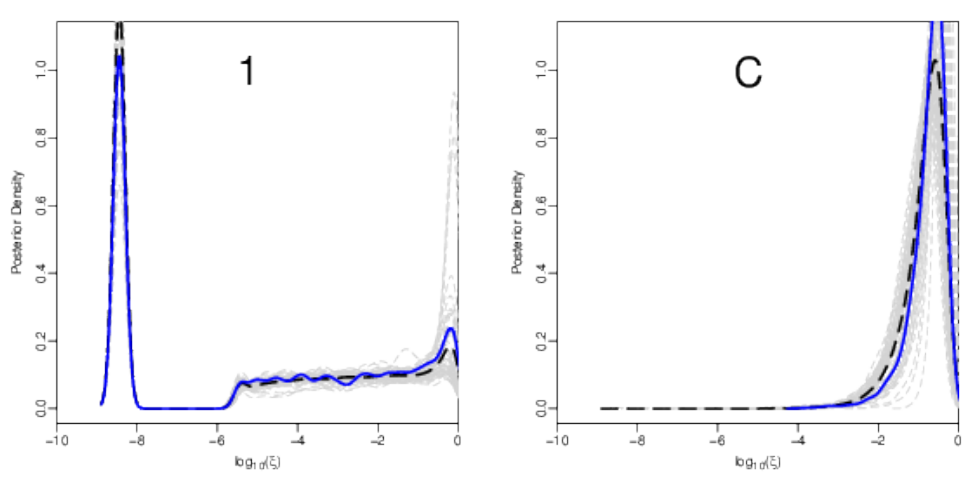

Fig. 10.- As in Figure 1, for quasar 1239+376 (ObsID 7871). 
(a)
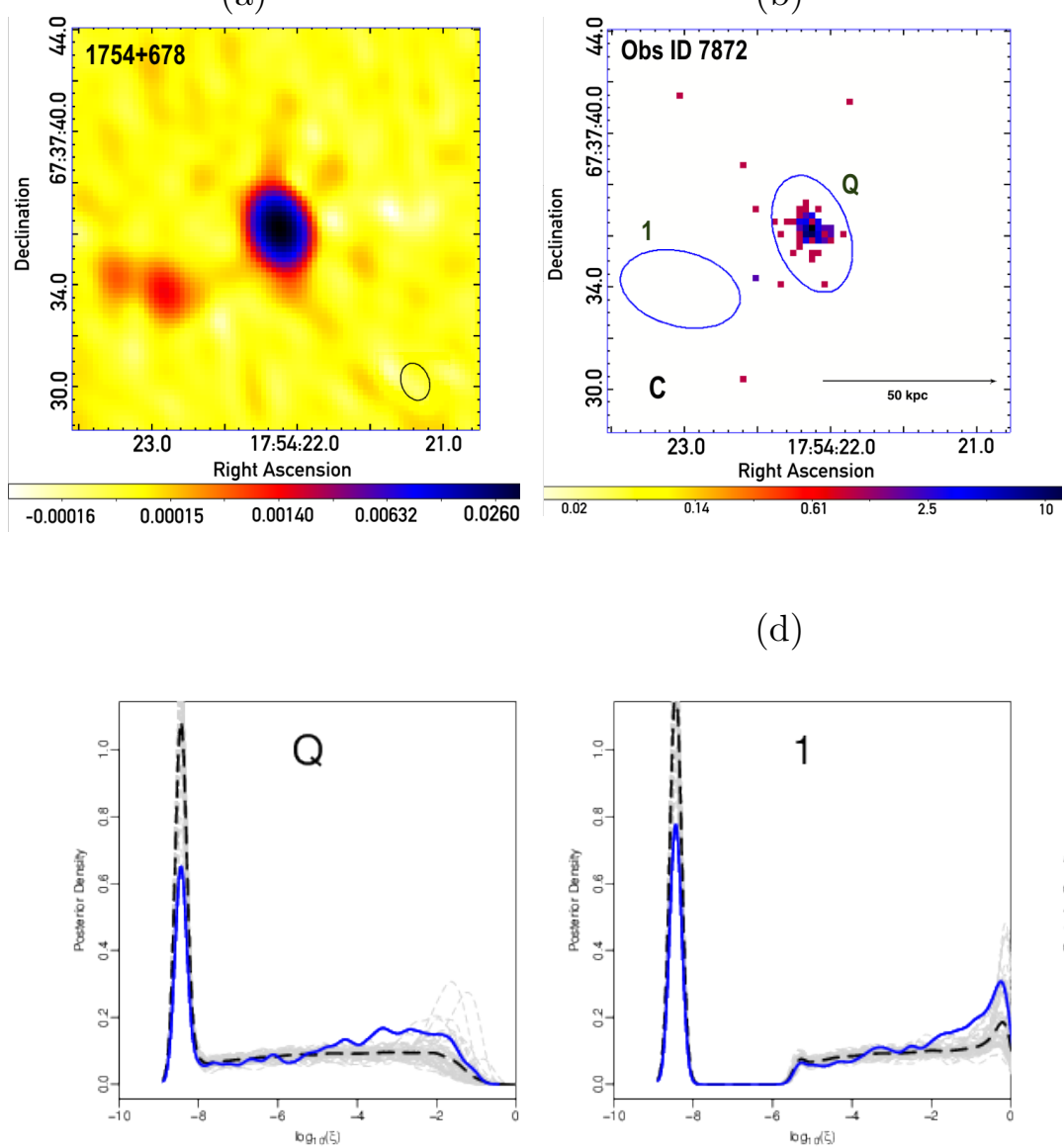

(d)

(b)

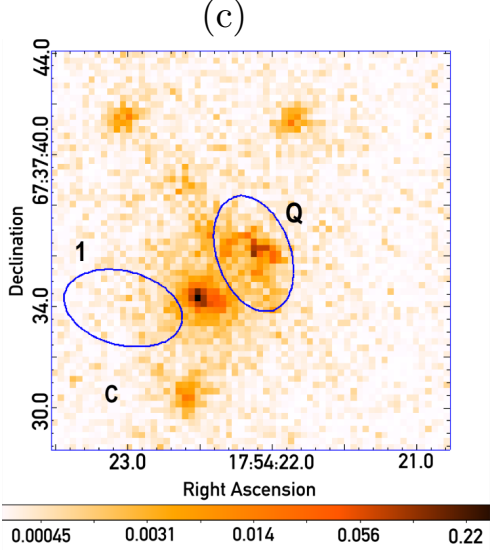

)
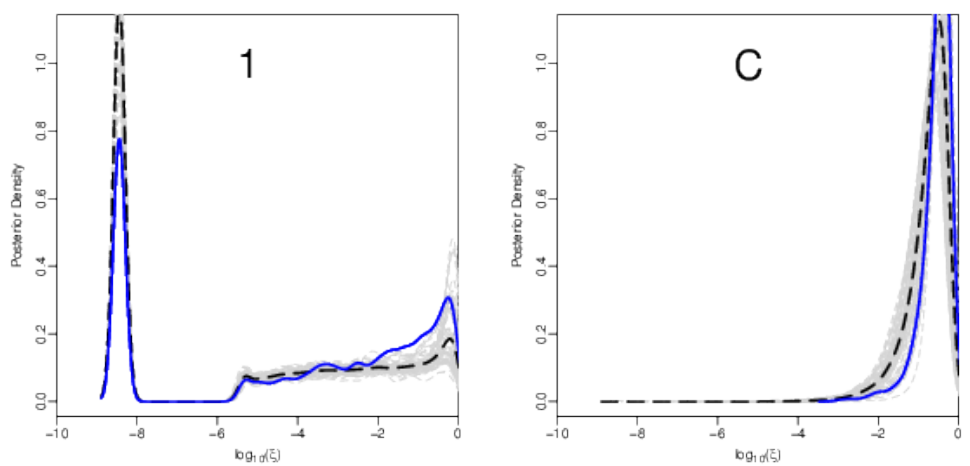

Fig. 11. - As in Figure 1, for quasar 1754+678 (ObsID 7872). 
(a)
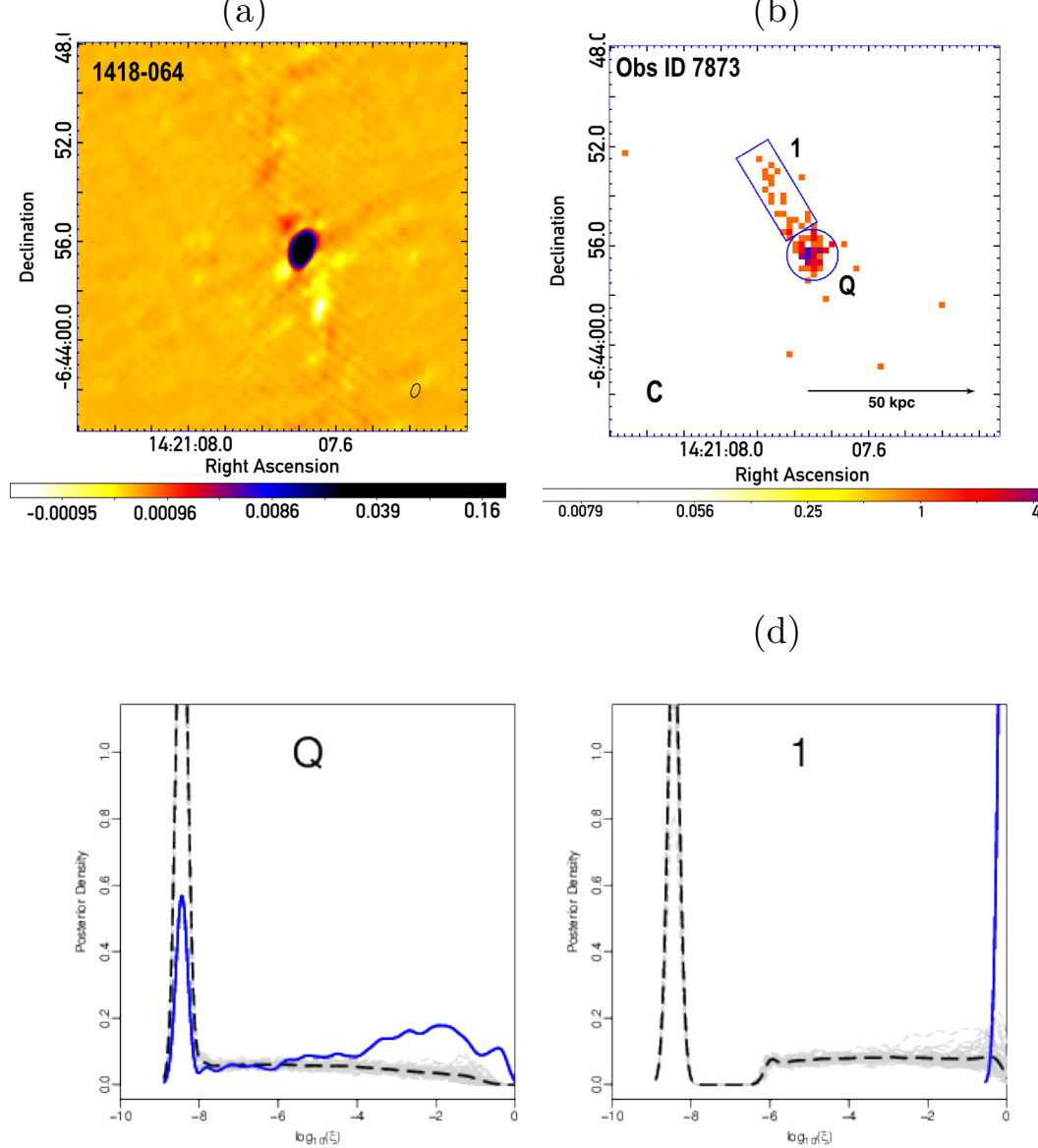

(d)

(b)

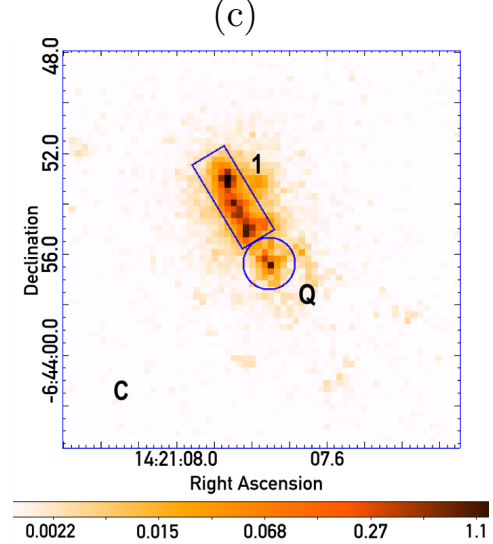

d)

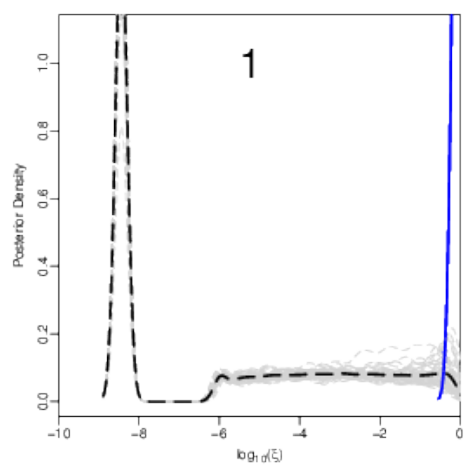

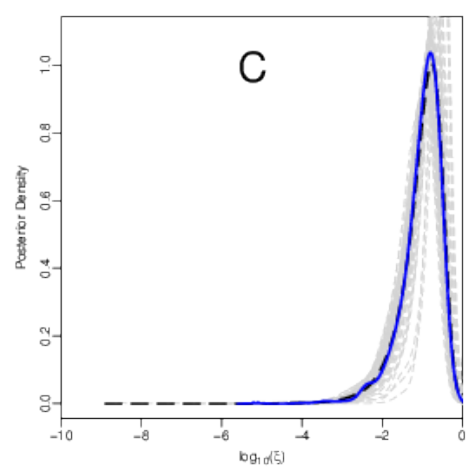

Fig. 12.- As in Figure 1, for quasar 1418-064 (ObsID 7873). 
(a)
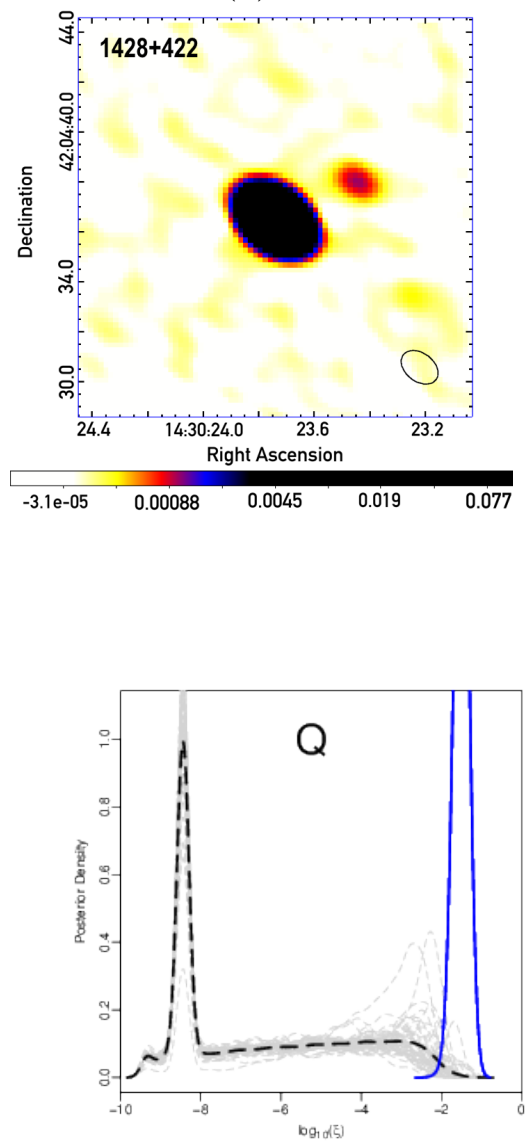

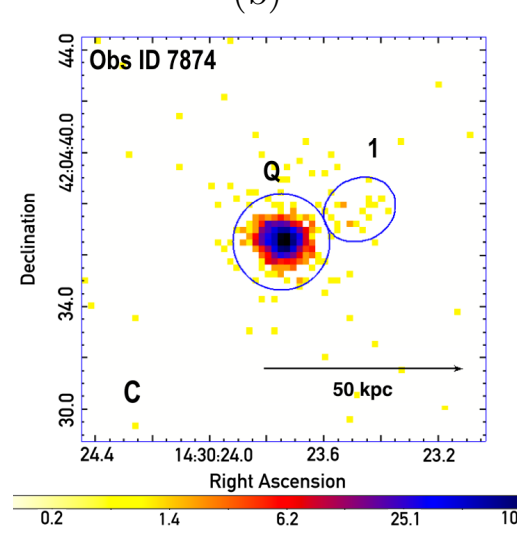

(d)

(b)

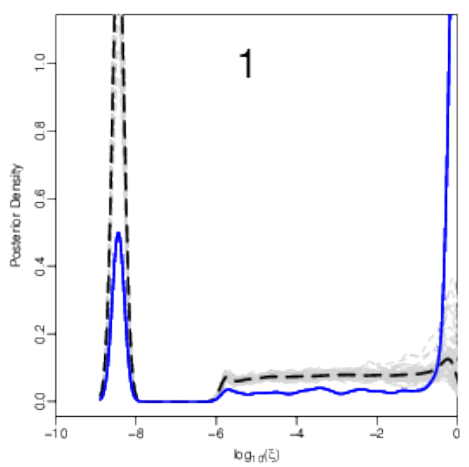

(c)
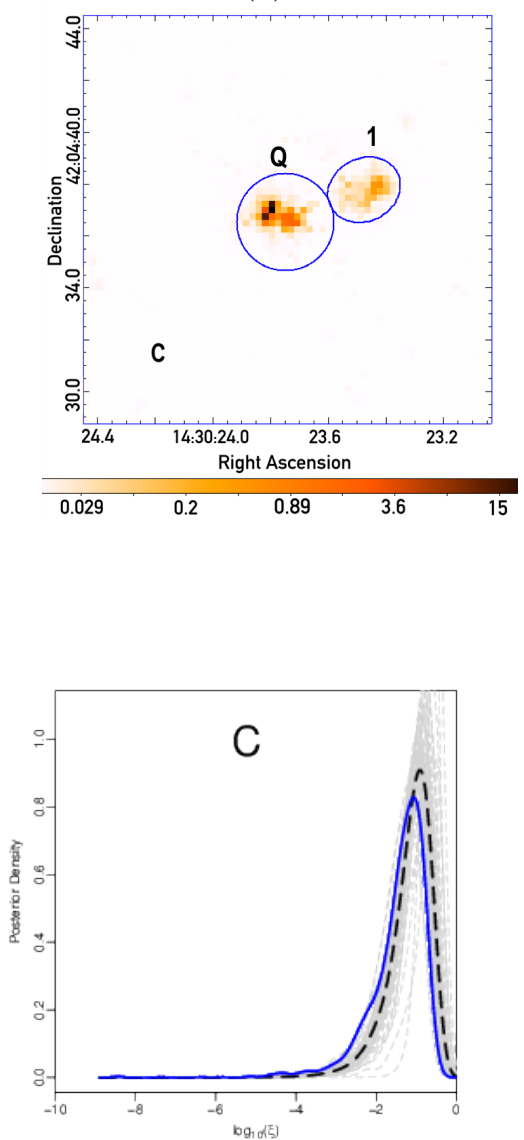

Fig. 13. - As in Figure 1, for quasar 1428+422 (ObsID 7874). 
(a)
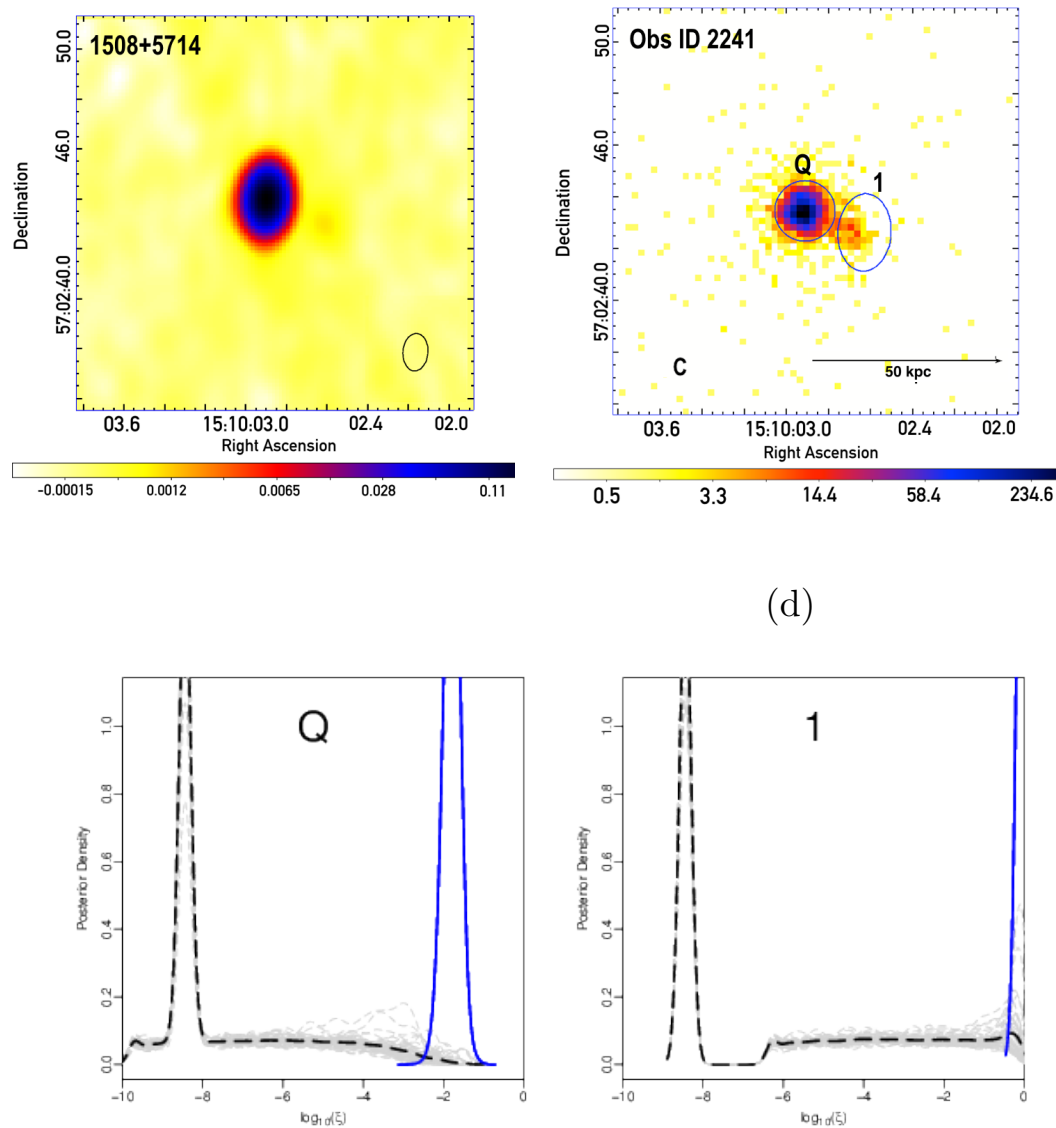

(d)

(b)

d)

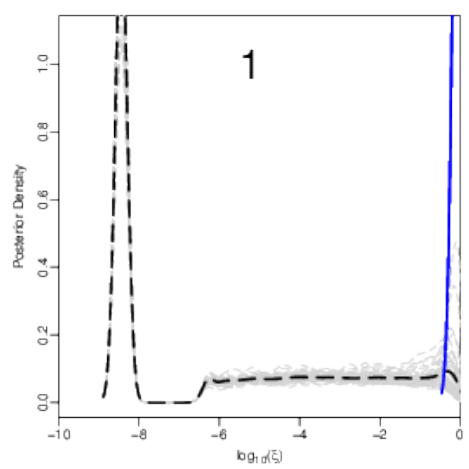

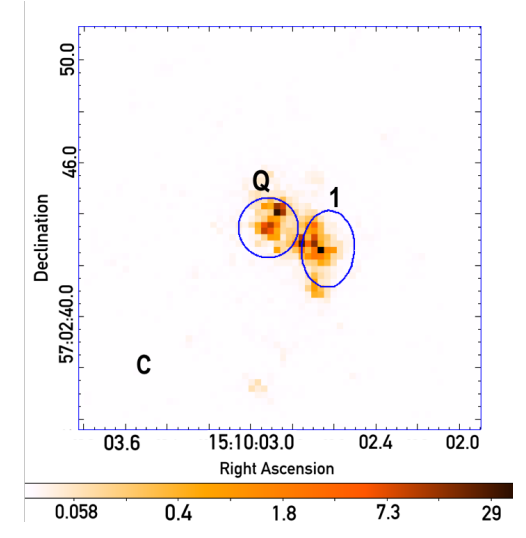

(c)

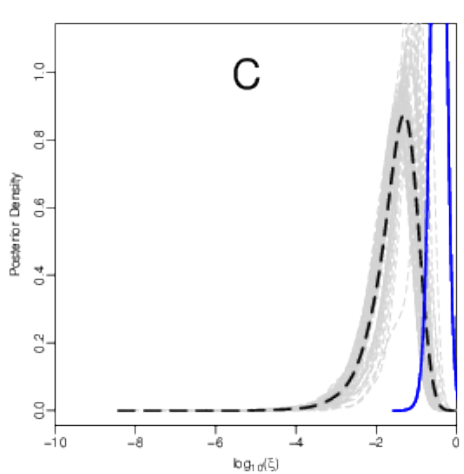

Fig. 14.- As in Figure 1, for quasar 1508+5714 (ObsID 2241). 